Volume 359, Number 12, December 2007, Pages 5697-5723

S 0002-9947(07)04102-5

Article electronically published on July 3, 2007

\title{
MARTIN POINTS ON OPEN MANIFOLDS OF NON-POSITIVE CURVATURE
}

\author{
JIANGUO CAO, HUIJUN FAN, AND FRANÇOIS LEDRAPPIER
}

\begin{abstract}
The Martin boundary of a Cartan-Hadamard manifold describes a fine geometric structure at infinity, which is a sub-space of positive harmonic functions. We describe conditions which ensure that some points of the sphere at infinity belong to the Martin boundary as well. In the case of the universal cover of a compact manifold with Ballmann rank one, we show that Martin points are generic and of full harmonic measure. The result of this paper provides a partial answer to an open problem of S. T. Yau.
\end{abstract}

\section{INTRODUCTION}

Let $\widetilde{M}$ be a Cartan-Hadamard manifold, a simply connected Riemannian manifold with non-positive curvature. Then, $\widetilde{M}$ is homeomorphic to an open ball, and there are two natural compactifications of $\widetilde{M}$ associated to the metric.

Fix $x_{0} \in \widetilde{M}$. For $z \in \widetilde{M}$, define the continuous function $b_{z}$ on $\widetilde{M}$ by

$$
b_{z}(x)=d(x, z)-d\left(x_{0}, z\right),
$$

where $d$ denotes the Riemannian distance on $\widetilde{M}$. The functions $b_{z}, z \in \widetilde{M}$ are equicontinuous and uniformly bounded on compact subsets of $\widetilde{M}$. They form a relatively compact set of functions for the topology of uniform convergence on compact sets. The closure of $\left\{z \mapsto b_{z}\right\}$ is the geometric compactification of $\widetilde{M}$. Let $\widetilde{M}(\infty)$ be the boundary of $\widetilde{M}$ in its geometric compactification. The set $\widetilde{M}(\infty)$, endowed with the relative topology, is homeomorphic to a sphere. Let $T(\widetilde{M})$ be the tangent bundle of $\widetilde{M}$, and $S_{x} \widetilde{M}=\left\{\vec{v} \in T_{x}(\widetilde{M}) \mid\|\vec{v}\|=1\right\}$ be the unit tangent sphere of $\widetilde{M}$ at $x$. For any $x \in \widetilde{M}$, the map $P_{x}: S_{x} \widetilde{M} \mapsto \widetilde{M}(\infty)$ which associates to $v \in S_{x} \widetilde{M}$ the point $P_{x} v=\sigma_{v}(+\infty)$ realizes this homeomorphism, where $\sigma_{v}$ is the geodesic with initial condition $v$ and, for a geodesic $\sigma$ in $\widetilde{M}$, we denote by $\sigma( \pm \infty)=\lim _{t \rightarrow+\infty} \sigma( \pm t)$ the corresponding points of $\widetilde{M}(\infty)$.

Assume that $\widetilde{M}$ admits a Green function $G(.,$.$) for the Laplace operator. For$ $z \in \widetilde{M}$, define the continuous function $h_{z}$ on $\widetilde{M}$ by

$$
h_{z}(x)=\log G(x, z)-\log G\left(x_{0}, z\right) .
$$

Received by the editors May 27, 2005.

2000 Mathematics Subject Classification. Primary 58J32; Secondary 60J65.

The first author was supported in part by an NSF grant.

The second author was partially supported by the Research Fund for returned overseas Chinese Scholars 20010107 and by the NSFC for Young Scholars 10401001. 
By Harnack's inequality, the functions $h_{z}, z \in \widetilde{M}$ are equicontinuous and uniformly bounded on compact subsets of $\widetilde{M}$ not containing $z$. They form a relatively compact set of functions for the topology of uniform convergence on compact sets. The closure of $\left\{z \mapsto h_{z}\right\}$ is the Martin compactification of $\widetilde{M}$.

For Euclidean spaces, the Martin compactification is reduced to the Alexandroff one-point compactification. If the sectional curvatures of $\widetilde{M}$ are pinched between two negative constants, then the Martin compactification coincides with the geometric compactification [AS]. In general, the presence of flats amidst negative curvature is a source of more intricate Martin compactification: for symmetric spaces, the Martin compactification has been described in [GJT] and is a non-trivial continuous extension of the geometric compactification; the general description of the Martin compactification of a product is not known in general; see [MV] and the references therein for the latest results. In these two cases, every geodesic belongs to a flat space. It is believed that, if many geodesics are not within a totally geodesic flat subspace, then the Martin compactification is the geometric compactification. See [Ba4] for a first example.

Following Ancona's programme (see $[\mathrm{An}]$ ), the same discussion applies to the general uniform elliptic operator $\mathcal{L}$ of second order in a general Cartan-Hadamard manifold $\widetilde{M}$ of dimension $n \geq 2$ with bounded geometry. The elliptic operator $\mathcal{L}$ has the form

$$
\mathcal{L}(u):=\operatorname{div}(\mathcal{A}(\nabla u))+B \cdot \nabla u+\operatorname{div}(u C)+\gamma u .
$$

The conditions for the coefficients will be given in the next section. If $\mathcal{L} u=0$, then $u$ is called an $\mathcal{L}$-harmonic function. We still denote by $G(\cdot, \cdot)$ the Green function of $\mathcal{L}$ and define $h_{z}(x)$ as before. Again fix $x_{0} \in \widetilde{M}$.

Definition 1.1 (Poisson kernel function). A Poisson kernel function $k_{\xi}(x)$ of $\mathcal{L}$ at $\xi \in \widetilde{M}(\infty)$ is a positive $\mathcal{L}$-harmonic function on $\widetilde{M}$ such that

$$
k_{\xi}\left(x_{0}\right)=1, k_{\xi}(y)=O\left(G_{x_{0}}(y)\right) \text { as } y \rightarrow \xi^{\prime} \neq \xi .
$$

Definition 1.2 (Martin point). We say that a point $\xi \in \widetilde{M}(\infty)$ is a Martin point of $\mathcal{L}$ if it satisfies the following properties:

- a) there exists a Poisson kernel function $k_{\xi}$ of $\mathcal{L}$ at $\xi$,

- b) the Poisson kernel function is unique, and

- c) if $y_{n} \rightarrow \xi$, then $h_{y_{n}} \rightarrow \log k_{\xi}$ uniformly on compact sets.

In this paper, we want to describe Martin points of $\mathcal{L}$ for Cartan-Hadamard manifolds. For that purpose, we introduce several local notions of negative curvature along a geodesic in $\widetilde{M}$. For a vector $v \in S \widetilde{M}$, the rank of $v$ is the dimension of the space of parallel Jacobi fields along the geodesic $\sigma_{v}$ with initial condition $v$. Clearly, $1 \leq \operatorname{rank} v \leq \operatorname{dim} \widetilde{M}$. The geodesic rank of the manifold $\widetilde{M}$ is the minimum value of $\{\operatorname{rank} v, v \in S \widetilde{M}\}$. For locally symmetric spaces, the geodesic rank coincides with the real rank of the real algebraic group of isometries of $\widetilde{M}$.

A geodesic $\sigma$ is called rank one if rank of $\sigma^{\prime}(0)$ is equal to 1 . A geodesic in $\widetilde{M}$ is called regular if it does not bound a totally geodesic flat half-space. Rank one geodesics are regular. In the next section, we introduce the notion of a hyperbolic at $\infty$ geodesic in $\widetilde{M}$. It is a precise qualitative property which expresses that the 
geodesic has an infinite number of segments surrounded by enough negative curvature. Geodesics in flats, or even geodesics converging to flats are not hyperbolic at $\infty$. Our main result is:

Theorem 1.3. Let $\widetilde{M}$ be a Cartan-Hadamard manifold with bounded geometry, $\mathcal{L}$ a uniformly elliptic, weakly coercive and bounded second order operator and $\sigma: \mathbb{R} \mapsto$ $\widetilde{M}$ a hyperbolic at $\infty$ geodesic. Then $\sigma(+\infty)$ is a Martin point of $\mathcal{L}$. In particular, if the Laplace operator $\Delta$ is weakly coercive, $\sigma(+\infty)$ is a Martin point for $\Delta$.

An axis in $\widetilde{M}$ is a geodesic which is invariant by an isometry of $\widetilde{M}$ with two fixed points at infinity. We will see that regular axes are hyperbolic at $\infty$.

Corollary 1.4. Let $\widetilde{M}$ be a Cartan-Hadamard manifold with bounded geometry, $\mathcal{L}$ a uniformly elliptic, weakly coercive and bounded second order operator and $\sigma$ : $\mathbb{R} \mapsto \widetilde{M}$ an axis such that $\sigma$ is not a boundary of any totally geodesic half-plane. Then $\sigma$ is hyperbolic at $\infty$ and $\sigma(+\infty)$ is a Martin point of $\mathcal{L}$.

Remark 1.5. If the sectional curvature of $\widetilde{M}$ is pinched, then Ancona ([An]) has proved that the Martin boundary $\partial_{\mathcal{L}} \widetilde{M}$ of $\widetilde{M}$ with respect to $\mathcal{L}$ is homeomorphic to the geometrical boundary $\widetilde{M}(\infty)$. Our result extends Ancona's results to nonpinched manifolds, at least at extremities of hyperbolic at $\infty$ geodesics.

In the rest of the paper, we show that if $\widetilde{M}$ is rank one and admits a cocompact group of isometries, then there are many hyperbolic at $\infty$ geodesics. So assume that the manifold $\widetilde{M}$ is the universal cover of a compact manifold $M$. Then, $\widetilde{M}$ has bounded geometry as soon as the metric is of class $C^{3}$, and the Laplace operator admits a Green function as soon as $M$ is not a 2-dimensional torus. Moreover, the geodesic rank rigidity results of Ballmann [Ba2] and Burns-Spatzier [BS] assert that $\widetilde{M}$ can be written uniquely as a product of Euclidean spaces, symmetric spaces and the universal covers of rank one spaces (see [K1], Appendix, for the existence of a cocompact action on the third factors). We shall therefore concentrate on rank one manifolds. We have:

Corollary 1.6. Let $\widetilde{M}$ be the universal cover of a compact Riemannian manifold of class $C^{3}$, non-positive curvature and geodesic rank $1, \mathcal{L}$ a uniformly elliptic, weakly coercive and bounded second order operator on $\widetilde{M}$ and $\sigma: \mathbb{R} \mapsto \widetilde{M}$ a regular axis. Then, $\sigma(+\infty)$ is a Martin point of $\mathcal{L}$. In particular, Martin points are dense in $\widetilde{M}(\infty)$.

Let $\Gamma=\pi_{1}(M)$ be the covering group. Recall that the action of $\Gamma$ by isometries on $\widetilde{M}$ extends to a continuous action on $\widetilde{M}(\infty)$. We set $(\widetilde{M}(\infty) \times \widetilde{M}(\infty))^{*}$ for the set of pairs of distinct points in $\widetilde{M}(\infty)$. We say that a finite positive measure $\mu$ on $\widetilde{M}(\infty)$ is geodesic ergodic if

- 1) The support of the measure $\mu \times \mu$ is $(\widetilde{M}(\infty) \times \widetilde{M}(\infty))^{*}$.

- 2) For $\mu \times \mu$ almost every $(\eta, \xi)$, there is a unique geodesic $\sigma_{\eta, \xi}$ such that $\sigma_{\eta, \xi}(-\infty)=\eta, \sigma_{\eta, \xi}(+\infty)=\xi$, and $\sigma_{\eta, \xi}$ is rank one.

- 3) The measure $\mu \times \mu$ is $\Gamma$ quasi-invariant and ergodic: the diagonal action of $\Gamma$ preserves the $(\mu \times \mu)$-negligible subsets of $(\widetilde{M}(\infty) \times \widetilde{M}(\infty))^{*}$; and all $\Gamma$-invariant measurable subsets of $(\widetilde{M}(\infty) \times \widetilde{M}(\infty))^{*}$ are either negligible or co-negligible. 
Examples of geodesic ergodic measures are the Patterson-Sullivan measure (see [K1]), other Gibbs measures constructed along the same lines, the harmonic measure for the Laplace operator on $\widetilde{M}$ (see [BL]), or analogously other harmonic measures associated to Markov equivariant symmetric operators on $\widetilde{M}$ or on $\Gamma$ ([Ka]). It is not known, even for surfaces, whether the visibility measure, obtained by projecting under $P_{x_{0}}$ the Lebesgue measure of the sphere $S_{x_{0}} \widetilde{M}$ is geodesic ergodic. We have

Theorem 1.7. Let $\widetilde{M}$ be the universal cover of a compact Riemannian manifold of class $C^{3}$, non-positive curvature and geodesic rank one, and $\mathcal{L}$ a uniformly elliptic, weakly coercive and bounded second order operator on $\widetilde{M}$. Then the set of Martin points is a generic subset of $\widetilde{M}(\infty)$ : it contains a countable intersection of open dense subsets. Moreover, the set of Martin points has full measure for any geodesic ergodic measure.

In the next section, we introduce the necessary definitions and present the general scheme of the proofs. In Section 3, we recall the potential theory of weakly coercive operators, and Section 4 contains the geometric properties of hyperbolic at $\infty$ geodesics we shall use. Theorem 1.3 reduces to Propositions we prove in Section 5, and Theorem 1.7 is proven in Section 6.

\section{Precise statements of Results And StRategy of the Proofs}

Let $\widetilde{M}$ be a complete Riemannian manifold of dimension $n \geq 2$. If $d(y, z)$ is sufficiently small, we let $\mathbb{P}_{y}^{z}$ denote the parallel transport from $y$ to $z$ along the unique length-minimizing geodesic segment.

We say that $\widetilde{M}$ has bounded geometry if there exists $r_{0}>0$ such that for any ball $B\left(x, r_{0}\right) \subset \widetilde{M}$, there exists a chart $\chi: B\left(x, r_{0}\right) \rightarrow \mathbb{R}^{n}$ satisfying a uniform first order quasi-isometry condition:

$$
\begin{aligned}
& C_{0}^{-1} d(y, z) \leq\left\|\left.D \chi_{x}\right|_{y}-\left.D \chi_{x}\right|_{z}\right\|^{*} \leq C_{0} d(y, z), \\
& \forall y, z \in B\left(x, r_{0}\right) \text {, with a constant } C_{0} \text { independent of } x,
\end{aligned}
$$

where

$$
\left\|\left.D \chi_{x}\right|_{y}-\left.D \chi_{x}\right|_{z}\right\|^{*}=\left\|\chi_{x}(y)-\chi_{x}(z)\right\|+\max _{\|\vec{v}\|=1}\left\{\left\|\left.\left(D \chi_{x}\right)\right|_{y} \vec{v}-\left.\left(D \chi_{x}\right)\right|_{z}\left(\mathbb{P}_{y}^{z} \vec{v}\right)\right\|\right\} .
$$

If the derivative of the curvature of $\widetilde{M}$ is bounded, and if the injectivity radius of $\widetilde{M}>0$, then (2.1) holds.

Consider the following elliptic operator $\mathcal{L}$ :

$$
\mathcal{L}(u):=\operatorname{div}(\mathcal{A}(\nabla u))+B \cdot \nabla u+\operatorname{div}(u C)+\gamma u,
$$

where $\mathcal{A}$ is a section of $\operatorname{End}(T \widetilde{M}), B$ and $C$ are vector fields on $\widetilde{M}$ and $\gamma$ is a function.

Definition 2.1. The operator $\mathcal{L}$ is called uniformly elliptic if there is a $\lambda>1 \mathrm{such}$ that

$$
\forall(x, u) \in T \widetilde{M}, \lambda^{-1}\|u\|^{2} \leq\left\langle\mathcal{A}_{x}(u), u\right\rangle \leq \lambda\|u\|^{2} .
$$

Definition 2.2. The operator $\mathcal{L}$ is said to be bounded if there is a $\lambda>0$ such that

$$
\forall x \in \widetilde{M},\|B\|_{L^{\infty}\left(B\left(x, r_{0}\right)\right)},\|C\|_{L^{\infty}\left(B\left(x, r_{0}\right)\right)},\|\gamma\|_{L^{\infty}\left(B\left(x, r_{0}\right)\right)} \leq \lambda .
$$


Definition 2.3. The function $G: \widetilde{M} \times \widetilde{M} \mapsto(0,+\infty)$ is called a Green function of $\mathcal{L}$ if $G$ is continuous and, for any $x \in \widetilde{M}, G_{x}(y):=G(y, x)$ is an $\mathcal{L}$-potential on $\widetilde{M}$ and is $\mathcal{L}$-harmonic on $\widetilde{M} \backslash\{x\}$ such that

$$
\mathcal{L}\left(G_{x}\right)=-\delta_{x}
$$

Definition 2.4. The operator $\mathcal{L}$ is called weakly coercive if there exists $\epsilon>0$ and a positive superharmonic function on $\widetilde{M}$ with respect to the operator $\mathcal{L}+\epsilon I$.

So if $\mathcal{L}$ is weakly coercive for some $\epsilon>0$, then for any $0 \leq t<\epsilon$, the operator $\mathcal{L}+t I$ has a Green function $G^{t}$.

Now let $\widetilde{M}$ be a Cartan-Hadamard manifold, and for $\sigma: \mathbb{R} \mapsto \widetilde{M}$ a geodesic line of unit speed, set

$$
\mathcal{U}_{h}(\sigma(\mathbb{R}))=\{y \mid d(y, \sigma(\mathbb{R}))=h\} .
$$

Since $\sigma(\mathbb{R})$ is a closed convex subset of $\widetilde{M}$, there exists a nearest-point projection: $\mathcal{P}_{\sigma}: \widetilde{M} \mapsto \sigma(\mathbb{R})$. We define

$$
S_{h}^{\perp}(\sigma(t))=\mathcal{P}_{\sigma}^{-1}(\sigma(t)) \cap \mathcal{U}_{h}(\sigma(\mathbb{R})),
$$

and

$$
\eta_{\sigma\left(\left[t_{1}, t_{2}\right]\right)}(h)=d_{\mathcal{U}_{h}}\left(S_{h}^{\perp}\left(\sigma\left(t_{1}\right)\right), S_{h}^{\perp}\left(\sigma\left(t_{2}\right)\right)\right),
$$

where $d_{\mathcal{U}_{h}}(\cdot, \cdot)$ is the distance function of the Riemannian hypersurface $\left(\mathcal{U}_{h},\left.g\right|_{\mathcal{U}_{h}}\right)$.

The following notion is a way of expressing at a finite distance that the geodesic $\sigma$ does not bound a flat half space:

Definition 2.5. A geodesic $\sigma: \mathbb{R} \mapsto \widetilde{M}$ is said to be $(h, T, \delta)$-non-flat at $t$ if we have:

$$
\eta_{\sigma([t, t+T])}(h)>T+\delta h .
$$

Properties of $(h, T, \delta)$-non-flat geodesics are recalled in Section 4. In particular, by Proposition 4.2 there exists a number $\varepsilon^{*}=\varepsilon^{*}(\widetilde{M}, h, T)$ such that if the geodesic $\sigma$ is $(h, T, \pi / 2)$-non-flat at 0 , and $\tau$ is another geodesic satisfying

$$
\tau(0)=\sigma(0) \text { and } \angle_{\sigma(0)}\left(\tau^{\prime}(0), \sigma^{\prime}(0)\right)<\varepsilon^{*},
$$

then the geodesic $\tau$ is $(h, T, \pi / 4)$-non-flat at 0 .

Let us now choose $\varepsilon^{*}<\pi / 4$ and set

$$
T_{1}=T_{1}(\widetilde{M}, h, T)=T+\frac{h}{\tan \varepsilon^{*}} .
$$

Definition 2.6. We say that the geodesic $\sigma$ admits an $(h, T, R)$ barrier if there exist $t_{i}, i=1,2, \ldots, 6$ with $T_{1}<t_{i+1}-t_{i}<T_{1}+R$ and $t_{3}+T<0<t_{4}$ such that the geodesic $\sigma$ is $(h, T, \pi / 2)$-non-flat at $t_{i}$, for $i=1,2, \ldots, 6$.

Remark 2.7. Observe that if a geodesic $\sigma$ is $(h, T, \pi / 2)$-non-flat at 0 , the geodesic $-\sigma$ obtained by reversing time is $(h, T, \pi / 2)$-non-flat at $-T$. Consequently, if the geodesic $\sigma$ admits an $(h, T, R)$ barrier, the geodesic $-\sigma$ admits an $(h, T, R)$ barrier as well, with $t_{i}^{\prime}=-t_{7-i}-T$.

Definition 2.8. We say that the geodesic $\sigma$ is hyperbolic at $\infty$ if there are $h, T, R$ and a sequence $t_{i}^{*} \rightarrow+\infty$ such that $\sigma\left(\cdot-t_{i}^{*}\right)$ admits an $(h, T, R)$ barrier. 
We have defined all elements of Theorem 1.3 that we recall:

Theorem 1.3. Let $\widetilde{M}$ be a Cartan-Hadamard manifold with bounded geometry, $\mathcal{L}$ a uniformly elliptic, weakly coercive and bounded second order operator and $\sigma$ : $\mathbb{R} \mapsto \widetilde{M}$ a hyperbolic at $\infty$ geodesic. Then $\sigma(+\infty)$ is a Martin point of $\mathcal{L}$.

In order to prove Theorem 1.3, we define the families of cones

$$
\Gamma_{\sigma, t, \theta}=\left\{x \in \widetilde{M} \mid \angle_{\sigma(t)}\left(\sigma^{\prime}(t), x\right)<\theta\right\} .
$$

Theorem 2.9. Suppose the geodesic $\tau$ admits an $(h, T, R)$ barrier. Set $T_{2}=$ $3\left(T_{1}+R\right)+T$. Then there is a constant $C=C(\widetilde{M}, h, T, R)$ such that the Green function $G(x, y)$ satisfies

$$
\begin{aligned}
& G(x, y) \leq C G(x, \tau(0)) G(\tau(0), y), \\
& \forall x \in \widetilde{M} \backslash \Gamma_{\tau,-T_{2}, \pi / 2}, \forall y \in \Gamma_{\tau, T_{2}, \pi / 2}
\end{aligned}
$$

and the Green function $g(x, y)$ in $\widetilde{M} \backslash \Gamma_{\tau, 2 T_{2}, \pi / 2}$ satisfies

$$
\begin{aligned}
& g(x, y) \leq C g(x, \tau(0)) g(\tau(0), y), \\
& \forall x \in \widetilde{M} \backslash \Gamma_{\tau,-T_{2}, \pi / 2}, \forall y \in \Gamma_{\tau, T_{2}, \pi / 2} \backslash \Gamma_{\tau, 2 T_{2}, \pi / 2} .
\end{aligned}
$$

Recall Definition 1.1 of a Poisson kernel function, and call $C_{\xi}$ the cone of functions positively proportional to a Poisson kernel function at $\xi \in \widetilde{M}(\infty)$. Then,

Proposition 2.10. Assume $\tau$ is a hyperbolic at $\infty$ geodesic with $\xi=\tau(+\infty)$. Then, $\operatorname{dim} C_{\xi} \leq 1$.

Proposition 2.11. Assume $\tau$ is a hyperbolic at $\infty$ geodesic with $\xi=\tau(+\infty)$, and consider the functions $k_{z}(x)=\frac{G(x, z)}{G\left(x_{0}, z\right)}$. Then, if $k_{\xi}$ is a limit point of $k_{z}$ as $z \rightarrow \xi$, $k_{\xi} \in C_{\xi}$.

Theorem 1.3 follows directly from Propositions 2.10 and 2.11. In Section 5, we prove Theorem 2.9 and explain how Propositions 2.10 and 2.11 follow from Theorem 2.9. In [An], (2.5) is called the Boundary Harnack Inequality and is a key step in the proof. For establishing (2.5), our task is to use as little negative curvature as we find necessary. The proof follows the ideas from [An], but given the delicate arguments involved, we prefer writing all the details. Then, following [An]'s scheme, Propositions 2.10 and 2.11 follow from Theorem 2.9. Our observation is that it is sufficient to have an infinite number of disjoint barriers converging to $\xi$, not necessarily a uniform estimate everywhere. Again we write the detailed proof for the sake of completeness.

Assume now that $\tilde{\sigma}: \mathbb{R} \mapsto \widetilde{M}$ is an axis and suppose that $\tilde{\sigma}$ is not the boundary of any totally geodesic half plane. Then, there exist $h_{0}$ and $\delta_{0}$ such that for any $k \in \mathbb{N}$, there is an integer $n$ such that $\tilde{\sigma}$ is $\left(h_{0}, n L, k \delta_{0}\right)$-non-flat at 0 , where $L$ is the period of axis $\tilde{\sigma}$.

Indeed, since $\tilde{\sigma}$ is invariant by an isometry, $\tilde{\sigma}$ is not the boundary of any totally geodesic flat two-dimensional quarter. Thus, by Corollary 4.4 there exist $T_{0}, h_{0}$ and $\delta_{0}$ such that

$$
\eta_{\tilde{\sigma}\left(\left[0, T_{0}\right]\right)}\left(h_{0}\right)-T_{0} \geq \delta_{0}>0 .
$$

Choose $n_{0}>T_{0} / L$ to be an integer. Thus, since the function $T \mapsto \eta_{\tau[0, T]}\left(h_{0}\right)-T$ is non-decreasing (see Proposition 4.1 and Proposition 4.3(5)):

$$
\eta_{\tilde{\sigma}\left(\left[0, n_{0} L\right]\right)}\left(h_{0}\right)-n_{0} L \geq \delta_{0} .
$$


For any integer $k$, we get, using semi-additivity (4.1) and the periodicity of $\tilde{\sigma}$,

$$
\eta_{\tilde{\sigma}\left(\left[0, k n_{0} L\right]\right)}\left(h_{0}\right)-k n_{0} L \geq k \delta_{0},
$$

which is the desired property by setting $n=n_{0} k$.

By invariance under isometries, the axis $\tilde{\sigma}$ is also $\left(h_{0}, n L, k \delta_{0}\right)$-non-flat at $K L$, for all $K \in \mathbb{N}$. By choosing $k$ such that $k \delta_{0}>\frac{\pi}{2} h_{0}$, and $t_{i}, i=1,2, \ldots, 6$ also multiples of $L$, we find a number $R$ such that the axis $\tilde{\sigma}$ admits an $\left(h_{0}, n L, R\right)$ barrier. By invariance by isometries again, the axis $\tilde{\sigma}$ is a hyperbolic at $\infty$ geodesic. Corollary 1.4 is therefore a particular case of Theorem 1.3.

Consider the case when the Cartan-Hadamard manifold $\widetilde{M}$ is the universal cover of a compact Riemannian manifold $M$ of geodesic rank one. Set $S M$ for the unit tangent bundle of $M$. A unit tangent vector $v \in S M$ is said to be regular, $(h, T, \delta)$ non-flat, admitting an $(h, T, R)$ barrier or hyperbolic at $\infty$ if any geodesic $\sigma_{\tilde{v}}$ defined by a lift $\tilde{v}$ of $v$ to $S \widetilde{M}$ has the same property. Ballmann ([Ba1]) showed that unit tangent vectors to regular closed geodesics are dense in $S M$. Therefore Corollary 1.6 directly follows from Theorem 1.4. The geodesic flow is a one parameter group $\varphi_{t}, t \in \mathbb{R}$ of diffeomorphisms of $S M$. There is a unique $\varphi$-invariant probability measure $\bar{\nu}$ on $S M$ which realizes the topological entropy. The measure $\bar{\nu}$ has full support on $S M$ and the geodesic flow is ergodic for $\bar{\nu}([\mathrm{K} 2])$. Therefore:

Proposition 2.12. Let $M$ be a compact Riemannian manifold of non-positive sectional curvature and geodesic rank 1 . Then the set of hyperbolic at $\infty$ unit tangent vectors contains a countable intersection of open dense sets in SM. Moreover, it has full measure for $\bar{\nu}$.

Proof. We know that a unit tangent vector to a regular closed geodesic admits an $(h, T, R)$ barrier for some $h, T$ and $R$. By Proposition 4.2, there is an open neighborhood $\mathcal{O}$ of such a unit vector $v$ such that all $v^{\prime} \in \mathcal{O}$ also admit an $(h, T, R)$ barrier. Since the measure $\bar{\nu}$ is ergodic and has full support, for all positive $K$ the set $\mathcal{O}_{K}$ of $v \in S M$ such that the geodesic ray $\sigma_{v}([K, \infty))$ intersects $\mathcal{O}$ is open dense in $S M$ and has full $\bar{\nu}$ measure. The set $\bigcap_{K} \mathcal{O}_{K}$ is a countable intersection of open dense sets of full $\bar{\nu}$ measure. By definition, any unit vector in $\bigcap_{K} \mathcal{O}_{K}$ is hyperbolic at $\infty$.

To prove Theorem 1.7, we still have to verify that the large set of unit vectors of Proposition 2.12 lifts and projects to a large subset of $\widetilde{M}(\infty)$. This relies on the properties of the measure $\bar{\nu}$ which have been established in [K2]; see Section 6 .

Remark 2.13. In the case when $\widetilde{M}$ is the universal cover of a compact rank 1 manifold, the Laplace operator $\Delta$ is weakly coercive (see below Section 3) and clearly uniformly elliptic and bounded. The conclusions of Corollary 1.6 and Theorem 1.7 hold for $\mathcal{L}=\Delta$.

\section{Preliminaries (Elliptic operators, Green functions AND THEIR ESTIMATES)}

Let $\widetilde{M}$ be a Cartan-Hadamard manifold with bounded geometry and $\mathcal{L}$ a uniformly elliptic, weakly coercive and bounded second order operator. Let $\mu$ be a positive measure on $\widetilde{M}$. Define $G \mu(x):=\int_{\widetilde{M}} G(x, y) d \mu(y)$. If $G \mu$ is not identically $+\infty$, then $G \mu$ is the only potential satisfying $\mathcal{L}(G \mu)=-\mu$. 
There are two important estimates (see $[\mathrm{An}])$ :

- For each $\omega=B\left(x, r_{0}\right) \subset \widetilde{M}$, and every $t, 0 \leq t \leq 1$, the Green function $g^{t}$ related to $\mathcal{L}+t I$ over $\omega$ satisfies

$$
g^{t}(y, z) \geq C, \forall y, z \in B\left(x, r_{0} / 2\right), \text { and } g^{t}(y, z) \leq C^{-1} \text { if } d(y, z) \geq \frac{r_{0}}{4},
$$

where $C=C(\mathcal{L})$ is independent of $x$ and $t$.

- (Harnack inequality) If $u>0$ is an $(\mathcal{L}+t I)$-harmonic function on $B\left(x, r_{0}\right)$, then

$$
C^{-1} u(x) \leq u(y) \leq C u(x),
$$

where $C=C(\mathcal{L})>0$.

The adjoint operator $\mathcal{L}^{*}$ of $\mathcal{L}$ is given by the formula:

$$
\mathcal{L}^{*}(u)=\operatorname{div}\left(\mathcal{A}^{*}(\nabla u)\right)-\operatorname{div}(B \cdot u)-C \cdot \nabla u+\gamma u .
$$

Note that the Green function ${ }^{*}(x, y)$ of $\mathcal{L}^{*}$ satisfies $\stackrel{*}{G}(x, y)=G(y, x)$.

Lemma 3.1 ([An], Lemma 1$)$. For each positive measure $\mu$ on $\widetilde{M}$ and each $t, 0 \leq$ $t<\epsilon$, we have

$$
G^{t}(\mu)=G(\mu)+G\left(G^{t}(\mu)\right)
$$

Let $\stackrel{*}{\mu}_{x}$ be the $\mathcal{L}^{*}$-harmonic measure of a point $x \in \Omega$, where $\Omega$ is a bounded region in $\widetilde{M}$. We have

Lemma 3.2 ([An], Lemma 3). Let $g$ be the $\mathcal{L}$-Green function of $\Omega$, and let $g_{x}(y)=0$ for $y \notin \Omega$. Then

$$
\mathcal{L}\left(g_{x}\right)=-\delta_{x}+\stackrel{*}{\mu} \text {. }
$$

Proof. We have the representation formula of $g(x, y)$ in terms of $G(x, y)$ and the harmonic measure $\stackrel{*}{\mu}_{x}$ :

$$
g_{x}(y)=\stackrel{*}{G}_{y}(x)-\int_{\partial \Omega} \stackrel{*}{G}_{y}(z) d \stackrel{*}{\mu}_{x}(z),
$$

for $x \in \Omega, y \in \widetilde{M}$. Then we have

$$
g_{x}=G_{x}-G\left(\mu_{x}^{*}\right)
$$

and so

$$
\mathcal{L}\left(g_{x}\right)=-\delta_{x}+\stackrel{*}{\mu}_{x}
$$

Denote by $g^{t}$ the $(\mathcal{L}+t I)$-Green function of $\Omega$, and by $\stackrel{*}{\mu}_{x}^{t}$ the $\left(\mathcal{L}^{*}+t I\right)$-harmonic measure of $x$ in $\Omega$. We have

Lemma 3.3 ([An], Lemma 4). If $0 \leq t<\epsilon, x \in \Omega$ and $g_{x} \leq k g_{x}^{t}$ for some $k>0$ and outside some compact subset of $\Omega$, then we have

$$
\stackrel{*}{\mu}{ }_{x} \leq k \stackrel{*}{\mu}{ }_{x}^{t}
$$


Definition 3.4. Let $\Omega$ be a not necessarily bounded region in $\widetilde{M}$. Letting $x \in \Omega$, the "reduit" of $G_{x}$ on $\bar{\Omega}^{c}$ is defined as

$$
R_{G_{x}}^{\bar{\Omega}^{c}}:=\inf \left\{s \mid s>0 \text { is } \mathcal{L} \text {-superharmonic on } \widetilde{M} \text {, and } s \geq G_{x} \text { on } \bar{\Omega}^{c}\right\} .
$$

This reduit is an $\mathcal{L}$-potential, and if we put $\nu_{x}=-\mathcal{L}\left(R_{G_{x}}^{\bar{\Omega}^{c}}\right)$, then $\forall z \in \widetilde{M} \backslash \bar{\Omega}$, we have the formula:

$$
\stackrel{*}{G}_{z}(x)=G_{x}(z)=\int G(z, y) d \nu_{x}(y),
$$

where $\nu_{x}$ is supported by $\partial \Omega$.

Proposition 3.5 ([An], Proposition 7). There is a constant $C=C(\widetilde{M}, \lambda, \epsilon)>0$ such that if $x, y \in \widetilde{M}$ and $d(x, y)=1$, then

$$
\frac{1}{C} \leq G^{t}(x, y) \leq C, \text { for } 0 \leq t<\epsilon
$$

Lemma 3.6 ([An], Lemma 9). There exists a constant $\delta=\delta(\widetilde{M}, \lambda, \epsilon), 0<\delta<1$, such that for each ball $B(x, 1)$ in $\widetilde{M}$, the $\mathcal{L}$-harmonic measure $\mu_{x}$ of $x$ in $B(x, 1)$ and the similar $(\mathcal{L}+\epsilon I)$-harmonic measure $\mu_{x}^{\epsilon}$ satisfy

$$
\mu_{x} \leq(1-\delta) \mu_{x}^{\epsilon}
$$

Proposition 3.7 ([An], Proposition 10). There are positive numbers $C$ and $\alpha$ such that

$$
G(x, y) \leq C e^{-\alpha d(x, y)} G^{\epsilon}(x, y), \forall x, y \in \widetilde{M}
$$

where $C$ and $\alpha$ depend only on $\widetilde{M}, \lambda$ and $\epsilon$.

Proof. By induction on $k \in \mathbb{N}, k \geq 1$, we prove that $G(x, y) \leq(1-\delta)^{k-1} G^{\epsilon}(x, y)$, for $d(x, y)=k$ and the $\delta$ given by Lemma 3.6.

When $k=1$, we have $G(x, y) \leq G^{\epsilon}(x, y)$, since $G^{\epsilon}(x, y)$ is an $\mathcal{L}$-superharmonic function.

Assume that the inequality holds for $d(x, y)=k$. We want to prove that it holds for $d(x, y)=k+1$. By the maximum principle, one has

$$
G_{x}(z) \leq(1-\delta)^{k-1} G_{x}^{\epsilon}(z), \forall z \in \widetilde{M} \backslash B(x, k)
$$

and, in particular, for $z \in \partial B(y, 1)$. Hence

$$
G_{x}(y)=\int_{\partial B(y, 1)} G_{x}(z) d \mu_{y}(z) \leq(1-\delta)^{k-1} \int G_{x}^{\epsilon}(z) d \mu_{y}(z) .
$$

Now by Lemma 3.6,

$$
G_{x}(y) \leq(1-\delta)^{k} \int G_{x}^{\epsilon}(z) d \mu_{y}^{\epsilon}(z)=(1-\delta)^{k} G_{x}^{\epsilon}(y) .
$$

This proves the proposition for $d(x, y)$ being an integer. The general case follows by the fact that $G_{x} \leq G_{x}^{\epsilon}$ and the Harnack inequality for $G_{x}^{\epsilon}$.

Remark 3.8. Let $\Omega=B(x, r)$. Then Proposition 3.7 holds for $G_{\Omega}$ and $G_{\Omega}^{\epsilon}$, with the constants $C, \alpha$ independent of $r$. This is because if we proved the estimate for $d(x, y) \leq r-1$, then if $r-1 \leq d(x, y)<r$, by the maximum principle, we would have

$$
G_{\Omega}(x, y) \leq C e^{-\alpha(r-1)} G_{\Omega}^{\epsilon}(x, y) .
$$


Remark 3.9. By the Harnack inequality and Proposition 3.5, it is easy to obtain the lower bound estimate of $G(x, y)$ :

$$
c e^{-\beta d(x, y)} \leq G(x, y),
$$

where $c, \beta>0$ only depend on the bounded geometry of $\widetilde{M}$ and the operator $\mathcal{L}$.

Corollary 3.10 ([An], Corollary 11). Given $\delta>0$, there exists $R=R(\widetilde{M}, \lambda, \epsilon, \delta)$ such that $\forall x \in \widetilde{M}$, and $\forall r \geq R$, the $\mathcal{L}$-harmonic measure $\mu_{x}$ of $x$ in $B(x, r)$ and the similar $(\mathcal{L}+\epsilon I)$-harmonic measure $\mu_{x}^{\epsilon}$ satisfy

$$
\mu_{x} \leq \delta \mu_{x}^{\epsilon}
$$

Proof. For given $\delta>0$, we can find $R=R(\widetilde{M}, \lambda, \epsilon, \delta)$ such that $C e^{-\alpha d(x, y)} \leq \delta$ for $y$ near $\partial B(x, r)$ for any $r \geq R$, where $C$ and $\alpha$ are from Remark 3.8. So

$$
G_{B}(x, y) \leq \delta G_{B}^{\epsilon}(x, y) \text {, for } y \text { near } \partial B(x, r),
$$

i.e.,

By Lemma 3.3, we have

$$
\stackrel{*}{G}_{B, x} \leq \delta \stackrel{*}{G} \underset{B, x}{\epsilon}
$$

$$
\mu_{x} \leq \delta \mu_{x}^{\epsilon}
$$

Assume now that the Cartan-Hadamard manifold $\widetilde{M}$ is cocompact, i.e., it is the universal cover of some compact Riemannian manifold $M$ with the lifted metric. Furthermore, we assume $M$ is of geodesic rank 1. It is known that the fundamental group $\pi_{1}(M)$ of $M$ contains a free group $F_{2}$, and hence $\pi_{1}(M)$ is non-amenable. By Brooks's result, the first eigenvalue of the Laplace operator

$$
\lambda_{1}(\widetilde{M})=\inf _{f \in H^{1,2}(\widetilde{M})} \frac{\int_{\widetilde{M}}|\nabla f|^{2}}{\int_{\widetilde{M}}|f|^{2}}>0 .
$$

Now let $G(x, y)$ be the Green function of the Laplace operator $\Delta$ on $\widetilde{M}$. Since $\widetilde{M}$ is cocompact, the sectional curvature $\left|K_{\widetilde{M}}\right|$ and its derivative are bounded and the injectivity radius $\operatorname{inj}(\widetilde{M})$ is positive. Thus $\widetilde{M}$ has the "bounded geometry" property (2.1). On the other hand, the Laplace operator $\Delta$ satisfies (2.3) and (2.4) obviously. If we can prove that $\Delta$ is weakly coercive, then all the conclusions in Section 2 hold for $\mathcal{L}=\Delta$ and its Green function.

Define the bilinear form

$$
a_{t}(u, \varphi)=\int_{\widetilde{M}}\langle\nabla u, \nabla \varphi\rangle-\int t\langle u, \varphi\rangle
$$

from $H^{1,2}(\widetilde{M}) \times H^{1,2}(\widetilde{M})$ to $\mathbb{C}$. The form $a_{t}(u, \varphi)$ is bounded, since

$$
\left|a_{t}(u, \varphi)\right| \leq\|u\|_{H^{1,2}} \cdot\|\varphi\|_{H^{1,2}},
$$

for $0 \leq t \leq 1$.

The form $a_{t}(u, \varphi)$ is coercive, since

$$
\begin{aligned}
a_{t}(u, u) & =\int|\nabla u|^{2}-t \int|u|^{2} \geq \int|\nabla u|^{2}-\frac{t}{\lambda_{1}-\delta} \int|\nabla u|^{2} \\
& \geq\left(1-\frac{t}{\lambda_{1}-\delta}\right) \frac{1}{\lambda_{1}-\delta} \int|u|^{2},
\end{aligned}
$$

for $0<\delta<\lambda_{1}, 0 \leq t<\lambda_{1}-\delta$. 
Hence

$$
a_{t}(u, u) \geq C_{\delta}\|u\|_{H^{1,2}(\widetilde{M})}^{2}
$$

for $0 \leq t<\lambda_{1}-\delta$, where

$$
C_{\delta}=\frac{1}{2}\left(1-\frac{t}{\lambda_{1}-\delta}\right) \min \left\{1, \frac{1}{\lambda_{1}-\delta}\right\}
$$

If we take $\delta=\frac{\lambda_{1}}{2}$, then for any $0 \leq t<\frac{\lambda_{1}}{3}$, there exists

$$
a_{t}(u, u) \geq C_{\lambda_{1}}\|u\|_{H^{1,2}}^{2}
$$

where $C_{\lambda_{1}}=\frac{1}{6} \min \left\{1, \frac{2}{\lambda_{1}}\right\}$. Now by the Lax-Milgram theorem, for any $f \in$ $H^{-1,2}(\widetilde{M})$, there exists a unique $u \in H^{1,2}$ such that

$$
a_{\lambda_{1} / 3}(u, v)=\langle f, v\rangle .
$$

Take $\varphi \geq 0, \varphi \in C_{0}^{\infty}(\widetilde{M})$. Then the above equality implies that

$$
\left(\Delta+\frac{\lambda_{1}}{3}\right) u=-\varphi \leq 0 .
$$

On the other hand,

$$
a_{\lambda_{1} / 3}\left(u^{-}, u^{-}\right)=a_{\lambda_{1} / 3}\left(-u, u^{-}\right)=-\int \varphi u^{-} \leq 0 .
$$

So by coercivity, there is $u^{-}=0$ and $u \geq 0$. Therefore if $\varphi \neq 0$, we obtain a positive superharmonic function $u>0$ of the operator $\Delta+\lambda_{1} / 3$.

Theorem 3.11. There exist two positive numbers $C$ and $\alpha$ depending only on the geometry of $M$ such that $\forall(x, y) \in \widetilde{M} \times \widetilde{M}$ and $d(x, y) \geq 1$, the following holds:

$$
G(x, y) \leq C e^{-\alpha d(x, y)} .
$$

Proof. This decay estimate was already proved in [SY]. Here we give a different proof. Firstly we prove that for $0<\epsilon<\lambda_{1} / 3$, and for any $x, y \in \widetilde{M}$ satisfying $d(x, y) \geq 1$, we have $G^{\epsilon}(x, y) \leq C$, where $C$ only depends on $\lambda_{1}$.

Let $f$ and $g$ be the characteristic function of the balls $B(x, \rho)$ and $B(y, \rho)$, respectively, where $\rho=\min \left\{r_{0}, 1 / 3\right\}$. Then $G^{\epsilon}(f d v)$ is the solution of the equation $\Delta u+\epsilon u=f$. By the Schwarz inequality and the Lax-Milgram theorem, we have

$$
\int G^{\epsilon}(f) \cdot g \leq\left(\int\left|G^{\epsilon}(f)\right|^{2}\right)^{\frac{1}{2}} \cdot\|g\|_{L^{2}} \leq C_{\lambda_{1} / 3}\|f\|_{L^{2}}\|g\|_{L^{2}}=C .
$$

Thus we have

$$
\iint_{(\xi, \eta) \in B(x, \rho) \times B(y, \rho)} G^{\epsilon}(\xi, \eta) d \xi d \eta \leq C .
$$

Therefore there exists a point pair $\left(x_{1}, y_{1}\right) \in B(x, \rho) \times B(y, \rho)$ such that

$$
G^{\epsilon}\left(x_{1}, y_{1}\right) \leq C \text {. }
$$

Using the Harnack inequality, we obtain

$$
G^{\epsilon}(x, y) \leq C
$$

for all $(x, y)$ such that $d(x, y) \geq 1$. Here $C$ only depends on $M$. By Proposition 3.7 , we are done. 
Corollary 3.12. Given $\delta>0$, there exists $R=R(M, \delta)$ such that $\forall x \in M$ and $r \geq R$, the $\Delta$-harmonic measure $\mu_{x}$ of $x$ in $B(x, r)$ and the similar $(\Delta+\epsilon)$-harmonic measure $\mu_{x}^{\epsilon}$ satisfy

$$
\mu_{x} \leq \delta \mu_{x}^{\epsilon}
$$

Proof. This is a direct conclusion from Corollary 3.10 and Theorem 3.11.

By Theorem 3.11, the Green function $G$ of the Laplace operator vanishes at infinity. For the Green function of the general elliptic operator $\mathcal{L}$, we need the following definition. Letting $\xi \in \widetilde{M}(\infty)$, we say that a function $u$ vanishes at $\xi$ in the $\mathcal{L}$-sense if there exists a positive $\mathcal{L}$-superharmonic function $w$ on $\widetilde{M}$ such that $u=o(w)$ at $\xi$. If $\mathcal{L}(1) \leq 0$, then the vanishing of $u$ at $\xi$ in the $\mathcal{L}$-sense is the same as usual. It is shown in [An], page 509, that for any $x \in \widetilde{M}, G_{x}$ vanishes on $\widetilde{M}$ in the $\mathcal{L}$-sense. Namely, there exists an $\mathcal{L}$-superharmonic function $w$ such that $G_{x}=o(w)$ at infinity.

Proposition 3.13. Let $0<\theta<\pi, \Gamma=\Gamma_{\sigma, t_{0}, \theta}$, and $\Gamma_{1}=\Gamma_{\sigma, t_{0}+T_{0}, \theta}$ for some $t_{0}$ and $T_{0}>0$. If $u(x)$ is a positive $\mathcal{L}$-harmonic function in $\Gamma$ and vanishes in the $\mathcal{L}$-sense in $\widetilde{M}(\infty) \cap \Gamma$, then the reduit $u_{1}(x):=R_{u}^{\Gamma_{1}}(x)$ is an $\mathcal{L}$-potential on $\Gamma$.

Proof. This is proved in [An], Theorem 2.

\section{Hyperbolicity estimates}

Let $\widetilde{M}$ be a Cartan-Hadamard manifold with bounded geometry, and recall the definition of $(h, T, \delta)$-non-flat geodesics. We have the following properties of the distance $\eta$ :

Proposition 4.1 (Semi-additivity). For any $h>0$, we have

$$
\eta_{\sigma\left(\left[t_{1}, t_{3}\right]\right)}(h) \geq \eta_{\sigma\left(\left[t_{1}, t_{2}\right]\right)}(h)+\eta_{\sigma\left(\left[t_{2}, t_{3}\right]\right)}(h)
$$

for $t_{1} \leq t_{2} \leq t_{3}$.

Proof. Let $\phi:\left[t_{1}, t_{3}\right] \mapsto \mathcal{U}_{h}$ be a path from $S_{h}^{\perp}\left(\sigma\left(t_{1}\right)\right)$ to $S_{h}^{\perp}\left(\sigma\left(t_{3}\right)\right)$. For clear topological reasons, the path $\phi$ must intersect $S_{h}^{\perp}\left(\sigma\left(t_{2}\right)\right)$ at $\phi\left(t^{*}\right)$. Let $L\left(\left.\phi\right|_{[s, s+\delta]}\right)$ be the length of $\left.\phi\right|_{[s, s+\delta]}$. We have

$$
L\left(\left.\phi\right|_{\left[t_{1}, t_{3}\right]}\right)=L\left(\left.\phi\right|_{\left[t_{1}, t^{*}\right]}\right)+L\left(\left.\phi\right|_{\left[t^{*}, t_{3}\right]}\right) \geq \eta_{\sigma\left(\left[t_{1}, t_{2}\right]\right)}(h)+\eta_{\sigma\left(\left[t_{2}, t_{3}\right]\right)}(h) .
$$

Proposition 4.2 (Continuity). For fixed $t_{1}, t_{2}$ and $h$, the function $\eta_{\sigma\left(\left[t_{1}, t_{2}\right]\right)}(h) d e$ pends continuously on $\sigma^{\prime}(0)$. Namely, for fixed $\delta_{0}$, there exists $\varepsilon=\varepsilon\left(\widetilde{M}, t_{1}, t_{2}, h, \delta_{0}\right)$ such that if $d_{S \widetilde{M}}\left(\sigma^{\prime}(0), \tau^{\prime}(0)\right)<\varepsilon$, then

$$
\left|\eta_{\sigma\left(\left[t_{1}, t_{2}\right]\right)}(h)-\eta_{\tau\left(\left[t_{1}, t_{2}\right]\right)}(h)\right|<\delta_{0} .
$$

Proof. Indeed if $\sigma^{\prime}(0)$ and $\tau^{\prime}(0)$ are close enough, then the closed sets

$$
\mathcal{U}_{h}\left(\sigma\left(\left[t_{1}, t_{2}\right]\right)\right), S_{h}^{\perp}\left(\sigma\left(t_{1}\right)\right) \text { and } S_{h}^{\perp}\left(\sigma\left(t_{2}\right)\right)
$$

are sufficiently close, to respectively, the closed sets

$$
\mathcal{U}_{h}\left(\tau\left(\left[t_{1}, t_{2}\right]\right)\right), S_{h}^{\perp}\left(\tau\left(t_{1}\right)\right) \text { and } S_{h}^{\perp}\left(\tau\left(t_{2}\right)\right)
$$

that the respective distances

$$
d_{\mathcal{U}_{h}}\left(S_{h}^{\perp}\left(\sigma\left(t_{1}\right)\right), S_{h}^{\perp}\left(\sigma\left(t_{2}\right)\right)\right) \text { and } d_{\mathcal{U}_{h}}\left(S_{h}^{\perp}\left(\tau\left(t_{1}\right)\right), S_{h}^{\perp}\left(\tau\left(t_{2}\right)\right)\right)
$$


are close. Moreover, by bounded geometry, if $t_{1}, t_{2}$ and $h$ are bounded, the explicit $\varepsilon$ of the above argument can be uniformly chosen, depending only on $\delta_{0}$.

The other properties of $\eta$ we use need to be made more explicit: Let $F=\exp$ : $\mathcal{N}(\sigma(\mathbb{R})) \mapsto \widetilde{M}$ be the exponential map (Fermi-map) along $\sigma$, where $\mathcal{N}(\sigma(\mathbb{R}))$ is the normal bundle along $\sigma$. If $\vec{Y}: \mathbb{R} \mapsto T_{\sigma} \widetilde{M}$ is a $C^{2}$-smooth vector field along $\sigma$ with $\vec{Y} \perp \sigma^{\prime}$ and $|\vec{Y}| \equiv 1$, we consider the map

$$
\begin{aligned}
F=F_{\vec{Y}}: \mathbb{R}_{+} \times\left[t_{1}, t_{2}\right] & \mapsto \widetilde{M} \\
(s, t) & \mapsto \exp _{\sigma(t)}(s \vec{Y}(t)) .
\end{aligned}
$$

For fixed $h$, the map $F(s, t)=\exp _{\sigma(t)}[s \vec{Y}(t)], \forall(s, t) \in[0, h] \times\left[t_{1}, t_{2}\right]$, gives a twodimensional embedding surface with image $\square_{t_{1}, t_{2}, h}$. Proposition 4.3 below implies that $F: \mathbb{R}^{2} \mapsto \widetilde{M}$ is a distance-increasing map, so the intrinsic curvature $K_{\square_{t_{1}, t_{2}, h}}$ is well defined. There is an intrinsic curvature function

$$
K_{\square_{t_{1}, t_{2}, h}}(s, t)=K\left(\frac{\partial F}{\partial t}, \frac{\partial F}{\partial s}\right)-\frac{\left|\nabla_{\frac{\partial F}{\partial s}} \frac{\partial F}{\partial t}\right|^{2}}{\left|\frac{\partial F}{\partial s} \wedge \frac{\partial F}{\partial t}\right|^{2}} .
$$

This curvature function is related to the following length function:

$$
l(h)=L\left(\left.F(h, \cdot)\right|_{\left[t_{1}, t_{2}\right]}\right)
$$

by the following proposition:

Proposition 4.3. Let $\sigma, \vec{Y}$ and $F=F_{\vec{Y}}$ be as above. Then

(1) $l(h)$ is a convex function of $h$.

(2) If $r(x)=d(x, \sigma(\mathbb{R}))$, then $\operatorname{Hess}(r)(X, X)=\left\langle\nabla_{X} \nabla r, X\right\rangle \geq 0$ and

$$
\frac{d l}{d h}=\int_{t_{1}}^{t_{2}}\left\langle\nabla_{\frac{\partial F}{\partial t}} \nabla r, \frac{\partial F}{\partial t}\right\rangle \frac{1}{\left|\frac{\partial F}{\partial t}\right|} d t=\int_{F(h, \cdot)} k_{g}(\cdot, h) d l \geq 0,
$$

where $k_{g}$ is the geodesic curvature of the curve $t \mapsto F(h, t)$ with respect to $\nabla r, k_{g}=-\left\langle\nabla_{\frac{\partial F}{\partial t}}\left(\frac{\partial F}{\partial t}\right), \nabla r\right\rangle=\operatorname{Hess}(r)\left(\frac{\partial F}{\partial t}, \frac{\partial F}{\partial t}\right)$.

(3)

$$
-\int_{\square_{t_{1}, t_{2}, h}} K_{\square_{t_{1}, t_{2}, h}} d A=\frac{\partial l}{\partial h} .
$$

$$
\frac{\partial l}{\partial h}(h) \geq \frac{l(h)-l(0)}{h} .
$$

$$
l(h) \geq t_{2}-t_{1} .
$$

Proof. Recall that $h \mapsto F(h, t)$ is a geodesic. Therefore

$$
J^{t}(h)=\frac{\partial F}{\partial t}(h, t)
$$

is a Jacobi field along the geodesic ray $\Psi_{t}: h \mapsto \Psi_{t}(h)=F(h, t)$.

It is easy to see that if $K_{\widetilde{M}} \leq 0$, then the function $h \mapsto\left\|J^{t}(h)\right\|$ is a convex function in $h$, i.e.,

$$
\frac{\partial^{2}\left\|J^{t}(h)\right\|}{\partial h^{2}} \geq 0
$$


Therefore

$$
\frac{\partial^{2} l}{\partial h^{2}}=\int_{t_{1}}^{t_{2}} \frac{\partial^{2}\left\|\frac{\partial F}{\partial t}\right\|}{\partial h^{2}} d t \geq 0
$$

For (2), this is a direct consequence of the first variational formula, where $\left.\nabla r\right|_{F(h, t)}$ $=\frac{\partial F}{\partial h}(h, t)$. In addition, it is proved in [BGS] that if $\sigma(\mathbb{R})$ is a convex subset, then $r(x)$ is a convex function in $x \in \widetilde{M}$.

The assertion (3) follows from the Gauss-Bonnet formula on $\square_{t_{1}, t_{2}, h}$. To see this we observe that $\|\vec{Y}(t)\|=1$. It is clear that $r(y) \equiv h$ for all $y \in \mathcal{U}_{h}(\sigma(\mathbb{R}))$. It follows that $r^{-1}(h)=\mathcal{U}_{h}(\sigma(\mathbb{R}))$ and $\left(\left.\nabla r\right|_{F(h, t)}\right) \perp \mathcal{U}_{h}(\sigma(\mathbb{R}))$. Hence, we have a rectangle of curved top.

The discussion above implies that

$$
\frac{\partial F}{\partial h}=\nabla r \perp \frac{\partial F}{\partial t}
$$

because $\frac{\partial F}{\partial t} \in T_{F(t, h)}\left[\mathcal{U}_{h}(\sigma(\mathbb{R}))\right]$.

Therefore, we apply the Gauss-Bonnet formula to get

$$
2 \pi=\frac{\pi}{2}+\frac{\pi}{2}+\frac{\pi}{2}+\frac{\pi}{2}+\int_{F(h, \cdot)} k_{g} d l+\int_{\square_{t_{1}, t_{2}, h}} K_{\square_{t_{1}, t_{2}, h}} d A .
$$

Thus,

$$
-\int_{\square_{t_{1}, t_{2}, h}} K_{\square_{t_{1}, t_{2}, h}} d A=\int_{F(h, \cdot)} k_{g} d l=\frac{\partial l}{\partial h} .
$$

This proves $(3)$.

For (4), we already proved that $l(h)$ is a convex function. Thus, we have

$$
\frac{\partial l}{\partial h} \geq \frac{l(h)-l(0)}{h} .
$$

Since, by (4.2), the left hand side of (3) is non-negative, $\frac{\partial l}{\partial h} \geq 0$ and so $l(h) \geq$ $l(0)=t_{2}-t_{1}$. This proves $(5)$.

By definition we have:

$$
\eta_{\sigma([t, t+T])}(h):=\inf _{\substack{|\vec{Y}|=1 \\ \vec{Y} \perp \sigma^{\prime}}}\left\{L\left(\left.F_{\vec{Y}}(h, \cdot)\right|_{[t, t+T]}\right)\right\} .
$$

Therefore, by Proposition 4.3, if a geodesic $\sigma$ is $(h, T, \delta)$-non-flat at $t$, then it satisfies:

$$
\hat{K}_{\sigma, h}(t, t+T):=\inf _{\substack{|\vec{Y}|=1 \\ \vec{Y} \perp \sigma^{\prime}}}\left\{\iint_{\square_{t, t+T, h}}-K_{\square_{t, t+T, h}}(s, t)\left|\frac{\partial F}{\partial s} \wedge \frac{\partial F}{\partial t}\right| d s d t\right\} \geq \delta .
$$

We also have:

Corollary 4.4. If for some $t, T$ and $h$ a geodesic $\sigma$ satisfies $\eta_{\sigma([t, t+T])}(h)=T$, then there is a field $\vec{Y}$ along $\sigma$ such that the rectangle $\square_{t, t+T, h}$ is totally geodesic and flat.

Proof. This assertion was indeed implicitly stated in [BGS]. For the convenience of the readers, we present a short proof here. Let $\mathcal{P}_{\sigma}: \widetilde{M} \mapsto \sigma(\mathbb{R})$ be the nearest point projection. Since $\widetilde{M}$ is a Cartan-Hadamard manifold and $\sigma(\mathbb{R})$ is a closed 
convex subset, it was proved in [BGS] that $\mathcal{P}_{\sigma}$ is a distance non-increasing map. Thus, we have

$$
d_{\widetilde{M}}(x, y) \geq d\left(\mathcal{P}_{\sigma}(x), \mathcal{P}_{\sigma}(y)\right)
$$

Equality holds in the above inequality if and only if the four points $\left\{x, y, \mathcal{P}_{\sigma}(x)\right.$, $\left.\mathcal{P}_{\sigma}(y)\right\}$ are vertices of a totally geodesic flat rectangle $\square$; see [BGS].

Suppose that $\eta_{\sigma([t, t+T])}(h)=T$. By compactness, there is a point $x \in S_{h}^{\perp}(\sigma(t))$, a point $y \in S_{h}^{\perp}(\sigma(t+T))$ and a shortest curve on $\mathcal{U}_{h}(\sigma([t, t+T]))$ realizing $d_{\mathcal{U}_{h}}(x, y)=$ $\eta_{\sigma([t, t+T])}(h)=T$. Therefore, we have the following equalities and inequalities:

$$
T=d_{\mathcal{U}_{h}}(x, y) \geq d_{\widetilde{M}}(x, y) \geq d\left(\mathcal{P}_{\sigma}(x), \mathcal{P}_{\sigma}(y)\right)=T .
$$

Hence, all inequalities above become equalities. In particular, we have $d_{\widetilde{M}}(x, y)=$ $d\left(\mathcal{P}_{\sigma}(x), \mathcal{P}_{\sigma}(y)\right)$, which implies that the four points $\left\{x, y, \mathcal{P}_{\sigma}(x), \mathcal{P}_{\sigma}(y)\right\}$ are vertices of a totally geodesic flat rectangle $\square_{t, t+T, h}$.

We can describe the geometric consequences of non-flatness we shall use. Let $\widetilde{M}$ be a Cartan-Hadamard manifold and $\sigma$ be a geodesic line of unit speed. Recall the family of cones $\Gamma_{\sigma, t, \theta}=\left\{x \in \widetilde{M} \mid \angle_{\sigma(t)}\left(\sigma^{\prime}(t), x\right)<\theta\right\}$.

Proposition 4.5. Suppose that the geodesic $\sigma$ is $(h, T, \pi / 4)$-non-flat at 0 . Then:

$$
\Gamma_{\sigma, T+h, 3 \pi / 4} \subset \Gamma_{\sigma, 0, \pi / 2} \text { and } \Gamma_{\sigma, T, \pi / 2} \subset \Gamma_{\sigma,-h, \pi / 4} .
$$

Proof. Let us show the first inclusion; the proof of the other one is similar. It suffices to show that there is no geodesic triangle with one side $\sigma([0, T+h])$, another side $\tau$ in $\partial \Gamma_{\sigma, T+h, 3 \pi / 4}$ and the third side in $\partial \Gamma_{\sigma, 0, \pi / 2}$. Suppose there is such a rectangle geodesic triangle $\triangle_{\sigma(0), \sigma(T+h), \tau(b)}$ with given three vertices $\{\sigma(T+h), \sigma(0), \tau(b)\}$, where $\tau(b) \in\left[\left(\partial \Gamma_{\sigma, T+h, 3 \pi / 4}\right) \cap\left(\partial \Gamma_{\sigma, 0, \pi / 2}\right)\right]$. We derive a contradiction as follows. We choose the vector field $\vec{Y}: \mathbb{R} \mapsto T_{\sigma} \widetilde{M}$ along $\sigma$ with $\vec{Y} \perp \sigma^{\prime}$ and $|\vec{Y}| \equiv 1$ in such a way that $\exp _{\sigma(t)}[S(t) \vec{Y}]$ lies in $\tau$ for some $S(t)$. As before, we let $\square_{0, T, h}=$ $\left\{\exp _{\sigma(t)}[s \vec{Y}] \mid 0 \leq t \leq T, 0 \leq s \leq h\right\}$. By comparison with the Euclidean plane, we have $S(t) \geq h$ for $0 \leq t \leq T$. Therefore the triangle $\triangle_{\sigma(0), \sigma(T+h), \tau(b)}=$ $\left\{\exp _{\sigma(t)}[s \vec{Y}] \mid 0 \leq t \leq T+h, 0 \leq s \leq S(t)\right\}$ contains the subset $\square_{0, T, h}$. By Proposition 4.3, (3)-(4), we have

$$
-\int_{\triangle_{\sigma(0), \sigma(T+h), \tau(b)}} K_{\triangle} d A \geq-\int_{\square_{t_{1}, t_{2}, h}} K_{\square_{t_{1}, t_{2}, h}} d A=\int_{F(h, \cdot)} k_{g} d l=\frac{\partial l}{\partial h}>\frac{\pi}{4} .
$$

This together with the Gauss-Bonnet formula implies that the sum of the inner angles of $\triangle_{\sigma(0), \sigma(T+h), \tau(b)}$ is smaller than $\left(\pi-\frac{\pi}{4}\right)=3 \pi / 4$, which is impossible.

The same proof also yields:

Proposition 4.6. Let $\varepsilon>0$, and suppose that there is $t_{+}, t_{-}>\frac{h}{\tan \varepsilon}$ such that the geodesic $\sigma$ is $(h, T, \pi / 2)$-non-flat at $t_{+}$and $-t_{-}$. Then

$$
\Gamma_{\sigma, T+t_{+}, \pi / 2} \subset \Gamma_{\sigma, 0, \varepsilon} \text { and } \Gamma_{-\sigma, t_{-}, \pi / 2} \subset \Gamma_{-\sigma,-T, \varepsilon} .
$$

The main geometric estimate related to the Martin boundary is Ancona's $\Phi$ chain condition. For a cone $\Gamma_{\sigma, 0, \theta}$, it says that one can find a time $T_{0}$ such that, for $x \in \partial \Gamma_{\sigma, 0, \theta}$,

$$
d\left(x, \Gamma_{\sigma, T_{0}, \theta}\right) \rightarrow \infty \text { as } d(x, \sigma(0)) \rightarrow \infty
$$


When $\widetilde{M}=\mathbb{R}^{n}$ is the Euclidean space, then for $x \in \partial \Gamma_{\sigma, 0, \theta}, d\left(x, \Gamma_{\sigma, T_{0}, \theta}\right) \leq T_{0}$ and can NOT be unbounded. For the same reason, if $\sigma(\mathbb{R})$ is a boundary of a totally geodesic flat half plane $\mathbb{R}_{+}^{2}$, then (4.7) fails on $\mathbb{R}_{+}^{2} \cap \partial \Gamma_{\sigma, 0, \theta}$. However, the cone property (4.5) implies a stronger form of (4.7).

Proposition 4.7. Let $0<\theta \leq \frac{\pi}{2}$. If $\Gamma_{\sigma, T_{0}, \theta+\varepsilon_{0}^{\prime}} \subset \Gamma_{\sigma, 0, \theta}$ for some $T_{0}>0$ and $\varepsilon_{0}^{\prime}>0$, then

$$
d\left(x, \Gamma_{\sigma, T_{0}, \theta}\right) \geq \varepsilon_{0}\left[d(x, \sigma(0))-\frac{1}{\varepsilon_{0}}\right]
$$

for $x \in \partial \Gamma_{\sigma, 0, \theta}$ and some $\varepsilon_{0}>0$ which depends only on $\varepsilon_{0}^{\prime}$ and $T_{0}$.

Proof. By our assumption, if $x \in \partial \Gamma_{\sigma, 0, \theta}$, then

$$
\angle_{\sigma\left(T_{0}\right)}\left(x, \Gamma_{\sigma, T_{0}, \theta}\right) \geq \varepsilon_{0}^{\prime} \text {. }
$$

Recall that $\exp _{\sigma\left(T_{0}\right)}: \mathbb{R}^{n} \mapsto \widetilde{M}$ is a distance increasing map. If

$$
\stackrel{\circ}{\Gamma}_{\sigma, T_{0}, \theta}=\left\{\vec{\omega} \in T_{\sigma\left(T_{0}\right)} \widetilde{M} \mid \angle\left(\vec{\omega}, \sigma^{\prime}\left(T_{0}\right)\right) \leq \theta\right\}
$$

and $\vec{u}_{x}=\exp _{\sigma\left(T_{0}\right)}^{-1} x$, then by (4.9) we have

$$
d_{\mathbb{R}^{n}}\left(\vec{u}_{x}, \stackrel{\circ}{\Gamma}_{\sigma, T_{0}, \theta}\right) \geq\left|\vec{u}_{x}\right| \sin \varepsilon_{0}^{\prime} .
$$

Since $\exp _{\sigma\left(T_{0}\right)}: \mathbb{R}^{n} \mapsto \widetilde{M}$ is distance increasing, we conclude that

$$
d_{\widetilde{M}}\left(x, \Gamma_{\sigma, T_{0}, \theta}\right) \geq d\left(x, \sigma\left(T_{0}\right)\right) \sin \varepsilon_{0}^{\prime} \geq\left[d(x, \sigma(0))-T_{0}\right] \sin \varepsilon_{0}^{\prime} .
$$

Then we choose $\varepsilon_{0}=\min \left\{\sin \varepsilon_{0}^{\prime}, \frac{1}{T_{0} \sin \varepsilon_{0}^{\prime}}\right\}$ and we obtain (4.8).

\section{Boundary Harnack Inequality and Martin boundary}

5.1. Boundary Harnack Inequality, proof of Theorem 2.9. We assume in this section that the geodesic $\tau$ admits an $(h, T, R)$ barrier, and we are going to prove (2.5). The proof of (2.6) is the same.

Proposition 5.1. Assume the geodesic $\tau: \mathbb{R} \mapsto \widetilde{M}$ is $(h, T, \pi / 4)$-non-flat at 0 and set $T_{0}=T+h$. Denote $x_{p}=\tau\left(p T_{0}\right), p \geq 0$. Then there exists a constant $C=C(\widetilde{M}, h, T)$ such that

$$
\begin{gathered}
G\left(y, x_{p}\right) \leq C G\left(x_{0}, x_{p}\right) G^{\epsilon}\left(y, x_{1}\right), \\
\forall y \in \widetilde{M} \backslash \Gamma_{\tau, 0, \pi / 2}, \forall p \geq 1 .
\end{gathered}
$$

Furthermore, for any $x \in \overline{x_{p} x_{p+1}}$, the line segment between $x_{p}$ and $x_{p+1}(p \geq 1)$, one has

$$
G(y, x) \leq C G\left(x_{0}, x\right) G^{\epsilon}\left(y, x_{1}\right), \forall y \in \widetilde{M} \backslash \Gamma_{\tau, 0, \pi / 2} .
$$

Proof. We denote $\Gamma=\Gamma_{\tau, 0, \pi / 2}, \Gamma_{1}=\Gamma_{\tau, T_{0}, \pi / 2}$.

To prove (5.1), we firstly prove the following inequality: there exists $C_{p}=$ $C(p, \widetilde{M}, h, T)$ such that

$$
G\left(y, x_{p}\right) \leq C_{p} G\left(x_{0}, x_{p}\right) G^{\epsilon}\left(y, x_{1}\right), \forall y \in \widetilde{M} \backslash \Gamma .
$$


By Remark 3.9, we have, with $C_{p}^{\prime \prime}=c e^{-\beta p T_{0}}$ for some $c, \beta$ depending only on $(\widetilde{M}, \mathcal{L})$ and

$$
G\left(x_{0}, x_{p}\right) \geq C_{p}^{\prime \prime}
$$

By construction, $B\left(x_{1}, h\right) \subset \Gamma$.

Take $y_{0} \in \partial B\left(x_{1}, h\right)$. Then by the Harnack inequality, we obtain

$$
G\left(y_{0}, x_{p}\right) \leq C_{p}^{\prime} G\left(y_{0}, x_{1}\right) .
$$

Applying the Harnack inequality to the variable $y$, we have

$$
G\left(y, x_{p}\right) \leq C_{p, 1}^{\prime} G\left(y, x_{1}\right), \forall y \in \partial B\left(x_{1}, h\right),
$$

with $C_{p, 1}^{\prime}=C_{p, 1}^{\prime}\left(\widetilde{M}, d\left(x_{1}, x_{p}\right)\right)$. Similarly we can prove that

$$
G\left(y, x_{p}\right) \leq C_{p, 2}^{\prime} G\left(y, x_{1}\right), \forall y \in \partial B\left(x_{p}, h\right),
$$

with $C_{p, 2}^{\prime}=C_{p, 2}^{\prime}\left(\widetilde{M}, d\left(x_{1}, x_{p}\right)\right)$. Let $C_{p}=\max \left\{C_{p, 1}^{\prime}, C_{p, 2}^{\prime}\right\}$. Combining (5.5) and (5.6), we have

$$
G\left(y, x_{p}\right) \leq C_{p} G\left(y, x_{1}\right), \forall y \in \partial B\left(x_{1}, h\right) \cup \partial B\left(x_{p}, h\right) .
$$

Now using the maximum principle, we have

$$
G\left(y, x_{p}\right) \leq C_{p} G\left(y, x_{1}\right), \forall y \in \widetilde{M} \backslash B\left(x_{1}, h\right) \cup B\left(x_{p}, h\right) .
$$

In particular, we have

$$
G\left(y, x_{p}\right) \leq C_{p} G\left(y, x_{1}\right) \leq C_{p} G^{\epsilon}\left(y, x_{1}\right), \forall y \in \widetilde{M} \backslash \Gamma .
$$

By (5.4) and (5.7), we obtain (5.3).

The proof of Proposition 5.1 will consist in showing that one can take the constant in (5.3) independent of $p$. Observe indeed that to obtain (5.3), we only used the relative distances of $x_{0}, x_{1}$ and $x_{p}$ and that $B\left(x_{1}, h\right) \subset \Gamma$. Therefore (5.3) can be applied to the cone $\Gamma_{1}$ to get

$$
G\left(y, x_{p+1}\right) \leq C_{p} G\left(x_{1}, x_{p+1}\right) G^{\epsilon}\left(y, x_{2}\right), \forall y \in \widetilde{M} \backslash \Gamma_{1} .
$$

Applying the Harnack inequality of $\mathcal{L}^{*}+\epsilon I$ to its Green function $G^{\epsilon}(y, x)$, one has

$$
G^{\epsilon}\left(y, x_{2}\right) \leq C^{\prime} G^{\epsilon}\left(y, x_{1}\right), \forall y \in \partial B\left(x_{2}, h\right) .
$$

Then the maximum principle and $B\left(x_{2}, h\right) \subset \Gamma_{1}$ imply that

$$
G^{\epsilon}\left(y, x_{2}\right) \leq C^{\prime} G^{\epsilon}\left(y, x_{1}\right), \forall y \in \widetilde{M} \backslash \Gamma_{1},
$$

where $C^{\prime}=C^{\prime}(\widetilde{M}, T+h)$. By the Harnack inequality, we have

$$
G\left(x_{1}, x_{p+1}\right) \leq C^{\prime \prime} G\left(x_{0}, x_{p+1}\right),
$$

where $C^{\prime \prime}=C^{\prime \prime}(\widetilde{M}, T+h)$ is independent of $p$ for $p \geq 1$.

Combining (5.8), (5.9) and (5.10), one has

$$
\begin{aligned}
G\left(y, x_{p+1}\right) & \leq C_{p} C^{\prime \prime} G\left(x_{0}, x_{p+1}\right) C^{\prime} G^{\epsilon}\left(y, x_{1}\right) \\
& =C_{p} C G\left(x_{0}, x_{p+1}\right) G^{\epsilon}\left(y, x_{1}\right), \forall y \in \widetilde{M} \backslash \Gamma_{1},
\end{aligned}
$$

where $C=C(\widetilde{M}, h, T)$. 
Now fixing $\delta=\frac{1}{C}$, by Corollary 3.10, $\forall \epsilon>0$, there exists $R_{0}=R_{0}(\widetilde{M}, h, T, \epsilon)$ such that $\forall x \in \widetilde{M}$ and $r \geq R_{0}$, the harmonic measures $\mu_{x}$ and $\mu_{x}^{\epsilon}$ on balls of radius $r$ about $x$ satisfy

$$
\mu_{x} \leq \delta \mu_{x}^{\epsilon}
$$

Since the geodesic $\tau$ is $(h, T, \pi / 4)$-non-flat at 0 , by Propositions 4.5 and 4.7 there is an $\varepsilon_{0}$ depending only on $T, h$ such that

$$
d\left(y, \partial \Gamma_{1}\right) \geq \varepsilon_{0}\left(d\left(y, x_{0}\right)-\frac{1}{\varepsilon_{0}}\right) .
$$

Now we can take $\rho_{1}=\rho_{1}\left(\varepsilon_{0}, R_{0}\right)=\rho_{1}(\widetilde{M}, h, T, \epsilon)$ such that for any $y \in \widetilde{M} \backslash \Gamma$ and $d\left(y, x_{0}\right) \geq \rho_{1}$ the following holds:

$$
d\left(y, \partial \Gamma_{1}\right) \geq R_{0} .
$$

For such a $y$, the ball $B\left(y, R_{0}\right) \subset \widetilde{M} \backslash \Gamma_{1}$. By (5.11), for any $z \in \partial B\left(y, R_{0}\right)$, we have

$$
G\left(z, x_{p+1}\right) \leq C_{p} C G\left(x_{0}, x_{p+1}\right) G^{\epsilon}\left(z, x_{1}\right) .
$$

So

$$
\begin{aligned}
& \int_{\partial B\left(y, R_{0}\right)} G\left(z, x_{p+1}\right) d \mu_{y}(z) \\
& \leq C_{p} C \int_{\partial B\left(y, R_{0}\right)} G^{\epsilon}\left(z, x_{1}\right) d \mu_{y}(z) G\left(x_{0}, x_{p+1}\right) \\
& \leq C_{p} C \delta \int_{\partial B\left(y, R_{0}\right)} G^{\epsilon}\left(z, x_{1}\right) d \mu_{y}^{\epsilon}(z) G\left(x_{0}, x_{p+1}\right) \\
& \leq C_{p} G\left(x_{0}, x_{p+1}\right) G^{\epsilon}\left(y, x_{1}\right),
\end{aligned}
$$

i.e.,

$$
G\left(y, x_{p+1}\right) \leq C_{p} G\left(x_{0}, x_{p+1}\right) G^{\epsilon}\left(y, x_{1}\right),
$$

for any $y \in \widetilde{M} \backslash \Gamma$ and $d\left(y, x_{0}\right) \geq \rho_{1}$.

There exists $C=C\left(\widetilde{M}, T_{0}\right)$ such that $G\left(x_{0}, x_{1}\right) C \geq 1$. Thus

$$
G\left(y, x_{p+1}\right) \leq C G\left(x_{0}, x_{p+1}\right) G\left(y, x_{1}\right)
$$

at $y=x_{0}$. Using Harnack's inequality in the compact set $(\widetilde{M} \backslash \Gamma) \cap B_{\rho_{1}}\left(x_{0}\right)$, one has

$$
G\left(y, x_{p+1}\right) \leq C^{\prime} G\left(x_{0}, x_{p+1}\right) G\left(y, x_{1}\right),
$$

for any $y \in(\widetilde{M} \backslash \Gamma) \cap B_{\rho_{1}}\left(x_{0}\right)$, where $C^{\prime}$ depends on $\rho_{1}$ and $C=C(\widetilde{M}, h, T)$ in (5.16), and hence depends only on $\widetilde{M}, h, T$, but not on the the integer $p$.

Combining (5.17) and (5.15), one obtains

$$
G\left(y, x_{p+1}\right) \leq \max \left\{C_{p}, C^{\prime}\right\} G\left(x_{0}, x_{p+1}\right) G^{\epsilon}\left(y, x_{1}\right) .
$$

So we can improve $C_{p+1}$ such that $C_{p+1}=\max \left\{C_{p}, C^{\prime}\right\}$.

Hence we can take a uniform constant $C=\max \left\{C_{1}, C^{\prime}\right\}$ such that for any $p \geq 0$ the following inequality holds:

$$
G\left(y, x_{p+1}\right) \leq C G\left(x_{0}, x_{p+1}\right) G^{\epsilon}\left(y, x_{1}\right), \forall y \in \widetilde{M} \backslash \Gamma \text { and } \forall p \geq 0,
$$

where $C$ depends only on $\widetilde{M}, h, T$. 
For the general point $x \in \overline{x_{p} x_{p+1}}$, one can use Harnack's inequality because of $d\left(x_{p}, x_{p+1}\right)=T_{0}$.

Recall from Section 2 the definition of $\varepsilon^{*}=\varepsilon^{*}(\widetilde{M}, h, T)$.

Corollary 5.2. Assume the geodesic $\sigma$ is $(h, T, \pi / 2)$-non-flat at 0 . Set $\Gamma=$ $\Gamma_{\sigma, 0, \pi / 2+\varepsilon^{*}}$ and $\Gamma_{1}=\Gamma_{\sigma, 0,+\varepsilon^{*}} \cap \Gamma_{\sigma, h+T, \pi / 2}$. Then, for any $y \in \widetilde{M} \backslash \Gamma$, for any $x \in \Gamma_{1}$, there is

$$
G(y, x) \leq C G\left(x_{0}, x\right) G^{\epsilon}\left(y, x_{1}\right),
$$

where $C=C(\widetilde{M}, h, T), x_{0}=\sigma(0)$, and $x_{1}=\sigma(h+T)$.

Proof. By our choice of $\varepsilon^{*}$ and Proposition 5.1, any geodesic $\tau$ which satisfies

$$
\tau(0)=\sigma(0) \text { and } \angle_{\sigma(0)}\left(\tau^{\prime}(0), \sigma^{\prime}(0)\right)<\varepsilon^{*}
$$

is $(h, T, \pi / 4)$-non-flat at 0 . By Proposition 5.1 for any $x \in \tau([h+T,+\infty))$ and any $y \in \widetilde{M} \backslash \Gamma_{\tau, 0, \pi / 2}$, we have

$$
G(y, x) \leq C G\left(x_{0}, x\right) G^{\epsilon}(y, \tau(h+T)),
$$

where $C=C(\widetilde{M}, h, T)$. On the other hand, by comparison $d\left(x_{1}, \tau(h+T)\right) \leq$ $\varepsilon^{*} \sinh (K(h+T))$, where $-K$ is a lower bound for the sectional curvature on $\widetilde{M}$, so that by Harnack's inequality, there is a $C=C(\widetilde{M}, h, T)$ such that for any $y \in \widetilde{M} \backslash \Gamma_{\tau, 0, \pi / 2}$ the following holds:

$$
G^{\epsilon}(y, \tau(h+T)) \leq C G^{\epsilon}\left(y, x_{1}\right) .
$$

Combining (5.19) and (5.20), we have the conclusion for any $x \in \Gamma_{\sigma, 0, \varepsilon^{*}}$ at distance at least $h+T$ from $\sigma(0)$ and any $y \in \widetilde{M} \backslash \bigcup_{\tau} \Gamma_{\tau, 0, \pi / 2}$, in particular for points $x \in \Gamma_{1}$ and $y \in \widetilde{M} \backslash \Gamma$.

By Remark 2.7, we can apply Corollary 5.2 to $-\sigma$ and get:

Corollary 5.3. Assume the geodesic $\sigma$ is $(h, T, \pi / 2)$-non-flat at 0 . Set $\Gamma^{\prime}=$ $\Gamma_{-\sigma,-T, \pi / 2+\varepsilon^{*}}$ and $\Gamma_{1}^{\prime}=\Gamma_{-\sigma,-T,+\varepsilon^{*}} \cap \Gamma_{-\sigma, h, \pi / 2}$. Then, for any $y \in \widetilde{M} \backslash \Gamma^{\prime}$, for any $x \in \Gamma_{1}^{\prime}$, we have

$$
G(y, x) \leq C G\left(x_{0}, x\right) G^{\epsilon}\left(y, x_{1}\right),
$$

where $C=C(\widetilde{M}, h, T), x_{0}=\sigma(T)$, and $x_{1}=\sigma(-h)$.

We can now prove Theorem 2.9:

Proof. Since the geodesic $\sigma$ admits an $(h, T, R)$ barrier, there are $t_{4}, t_{5}$, with $h / \tan \varepsilon^{*}$ $\leq t_{5}-t_{4} \leq T_{1}+R$ such that $\sigma$ is $(h, T, \pi / 2)$-non-flat at $t_{4}$. By Proposition 4.6, then

$$
\Gamma_{-\sigma,-t_{4}, \pi / 2} \subset \Gamma_{-\sigma,-t_{5}-T, \varepsilon^{*}} .
$$

Moreover, there is $t_{6}$, with $T+\frac{h}{\tan \epsilon^{*}} \leq t_{6}-t_{5} \leq R$ such that $\sigma$ is $(h, T, \pi / 2)$-non-flat at $t_{6}$ and by Proposition 4.6,

$$
\Gamma_{\sigma, t_{6}+T, \pi / 2} \subset \Gamma_{\sigma, t_{5}+T, \varepsilon^{*}}
$$

Applying Corollary 5.3 we get for any $y \in \Gamma_{\sigma, t_{6}+T, \pi / 2}$ and for any $x \in \widetilde{M} \backslash$ $\Gamma_{\sigma, t_{4}, \pi / 2}$,

$$
G(y, x) \leq C_{1} G\left(\sigma\left(t_{5}+T\right), x\right) G^{\epsilon}\left(y, \sigma\left(t_{5}-h\right)\right)
$$


where $C_{1}=C_{1}(\widetilde{M}, h, T)$. Using Harnack's inequality, we have with a different $C_{1}=C_{1}(\widetilde{M}, h, T)$ :

$$
G(y, x) \leq C_{1} G\left(\sigma\left(t_{6}+T\right), x\right) G^{\epsilon}(y, \sigma(0)) .
$$

In the same way, using that the geodesic $\sigma$ is $(h, T, \pi / 2)$-non-flat at $t_{1}, t_{2}$ and $t_{3}$, and Corollary 5.2, we can obtain that for any $x \in \widetilde{M} \backslash \Gamma_{\sigma, t_{1}, \pi / 2}$ and for any $y \in \Gamma_{\sigma, t_{3}+T, \pi / 2}$,

$$
G(x, y) \leq C_{2} G^{\epsilon}\left(x, \sigma\left(t_{2}+T+h\right)\right) G\left(\sigma\left(t_{2}\right), y\right)
$$

and

$$
G(x, y) \leq C_{2} G^{\epsilon}(x, \sigma(0)) G\left(\sigma\left(t_{1}\right), y\right),
$$

where $C_{2}=C_{2}(\widetilde{M}, h, T)$.

Set $x_{0}=\sigma\left(t_{1}\right), x^{\prime}=\sigma(0)$ and $x_{1}=\sigma\left(t_{6}+T\right)$, and let $\Gamma=\Gamma_{\sigma, t_{1}, \pi / 2}, \Gamma^{\prime}=\Gamma_{\sigma, 0, \pi / 2}$ and $\Gamma_{1}=\Gamma_{\sigma, t_{6}+T, \pi / 2}$. We claim that the cone pair $\left\{\Gamma, \Gamma_{1}\right\}$ satisfies the conclusion of Theorem 2.9. Since $-T_{2} \leq t_{1}$ and $t_{6}+T \leq T_{2}$, Theorem 2.9 will follow.

We follow [An]. Let $y \in \widetilde{M} \backslash \Gamma$ and $x \in \Gamma_{1}$. We have the representation

$$
\stackrel{*}{G}_{y}(x)=G(y, x)=\int_{\partial \Gamma^{\prime}} G(y, z) d \mu_{x}(z),
$$

where $\mu_{x}$ is a positive measure supported on $\partial \Gamma^{\prime}$ and such that

$$
G\left(\mu_{x}\right)=R_{G_{x}}^{\partial \Gamma^{\prime}}=R_{G_{x}}^{\omega}, \omega=\widetilde{M} \backslash \bar{\Gamma}^{\prime} .
$$

Now applying inequality $(5.22)$ to $\stackrel{*}{G}$, we have

$$
\begin{aligned}
G(y, x) & \leq C \int_{\partial \Gamma^{\prime}} G\left(y, x^{\prime}\right) G^{\epsilon}\left(x_{0}, z\right) d \mu_{x}(z) \\
& \leq C G\left(y, x_{1}\right) \int_{\partial \Gamma^{\prime}} G_{x_{0}}^{*}(z) d \mu_{x}(z) .
\end{aligned}
$$

Since $G^{\epsilon}\left(x_{0}, z\right)=\stackrel{*}{G_{x_{0}}^{\epsilon}}(z)$ is an $\mathcal{L}^{*}$-potential, so $\stackrel{*}{G}_{x_{0}}^{\epsilon}(z)=\stackrel{*}{G}(\lambda)$ for some positive measure $\lambda$ on $\widetilde{M}$. We have

$$
\int_{\partial \Gamma^{\prime}} \stackrel{*}{G}_{x_{0}}^{\epsilon}(z) d \mu_{x}(z)=\int_{\widetilde{M}} \int_{\partial \Gamma^{\prime}} G(y, z) d \mu_{x}(z) d \lambda(y)=\int_{\widetilde{M}} G\left(\mu_{x}\right) d \lambda(y) .
$$

By inequality (5.24), for any $z \in \partial \Gamma^{\prime}$, we have

$$
G_{x}(z) \leq C G_{x}\left(x^{\prime}\right) G_{x_{1}}^{\epsilon}(z) \leq C G_{x}\left(x_{0}\right) G_{x_{1}}^{\epsilon}(z) .
$$

By the definition of reduit, we have by (5.27),

$$
G\left(\mu_{x}\right)=R_{G_{x}}^{\partial \Gamma^{\prime}} \leq G_{x} \leq C G\left(x_{0}, x\right) G_{x_{1}}^{\epsilon} .
$$

Substituting (5.28) into (5.26) and then plugging in (5.25), we can obtain

$$
G(y, x) \leq C G\left(y, x_{1}\right) G\left(x_{0}, x\right) \int_{\widetilde{M}} G_{x_{1}}^{\epsilon} d \lambda .
$$

Now we only need to show that $\int_{\widetilde{M}} G_{x_{1}}^{\epsilon} d \lambda$ is bounded. By Lemma 3.1, we have

$$
\stackrel{*}{G}_{x_{0}}^{\epsilon}=\stackrel{*}{G} x_{x_{0}}+\epsilon \stackrel{*}{G}\left(\stackrel{*}{G} x_{x_{0}}^{\epsilon}\right) \text {. }
$$

Since $\stackrel{*}{G_{x_{0}}^{\epsilon}}=\stackrel{*}{G}(\lambda)$, so

$$
\lambda=-\mathcal{L}^{*}\left(\stackrel{*}{G} x_{x_{0}}\right)=\delta_{x_{0}}+\epsilon \stackrel{*}{G}_{x_{0}}^{\epsilon} d v_{\widetilde{M}}
$$


Thus

$$
\begin{aligned}
\int \stackrel{*}{G}_{x_{1}}^{\epsilon} d \lambda(z) & =G^{\epsilon}\left(x_{1}, x_{0}\right)+\epsilon \int G^{\epsilon}\left(z, x_{1}\right) G^{\epsilon}\left(x_{0}, z\right) d v_{\widetilde{M}}(z) \\
& =G^{\epsilon}\left(x_{1}, x_{0}\right)+\epsilon \stackrel{*}{G}^{\epsilon}\left(\stackrel{*}{G}{ }_{x_{1}}\right)\left(x_{0}\right) \\
& =\stackrel{*}{G}_{x_{1}}^{\epsilon}\left(x_{0}\right)+\epsilon \stackrel{*}{G}^{\epsilon}\left(\stackrel{*}{G_{x_{1}}^{\epsilon}}\right)\left(x_{0}\right) .
\end{aligned}
$$

Using Lemma 3.1 again to the operator $\mathcal{L}^{*}+\epsilon I$ and $\frac{\epsilon}{2}$ instead of $\mathcal{L}$ and $\epsilon$, we have

$$
\stackrel{*}{G}_{x_{1}}^{\epsilon}\left(x_{0}\right)+\epsilon \stackrel{*}{G}^{\epsilon}\left(\stackrel{*}{G} x_{x_{1}}^{\epsilon}\right)\left(x_{0}\right) \leq \widehat{G}\left(x_{0}, x_{1}\right) \leq C,
$$

where $\widehat{G}(x, y)$ is the Green function of $\mathcal{L}^{*}+\frac{\epsilon}{2}$. By Proposition 3.5, the constant $C$ here only depends on $M, \mathcal{L}$ and the distance between $x_{0}$ and $x_{1}$ and hence depends only on $M, \mathcal{L}, \theta, \tau$.

Combining (5.29), (5.30) and (5.31), the proof is finished.

\subsection{Proof of Proposition 2.10.}

Proof. Let $u(x), v(x) \in C_{\xi}$. Since $v(x)=O\left(G_{x_{0}}\right)=o(w)$, for some $\mathcal{L}$-superharmonic function, for $x \in \bar{\Gamma}_{-\tau,-t_{k}-2 T_{2}, \pi / 2} \cap \widetilde{M}(\infty)$, the reduit $v_{1}(x)$ of $v(x)$ on $\Gamma_{-\tau,-t_{k}-T_{2}, \pi / 2}$ with respect to $\Gamma_{-\tau,-t_{k}-2 T_{2}, \pi / 2}$ is a potential by Proposition 3.13. So it has the representation formula:

$$
v_{1}(x)=\int_{\partial \Gamma_{-\tau,-t_{k}-T_{2}, \pi / 2}} g^{k}(x, y) d \nu^{k}(y), \forall x \in \Gamma_{-\tau,-t_{k}+T_{2}, \pi / 2},
$$

where $\nu_{k}(y)$ is a positive measure on $\partial \Gamma_{-\tau,-t_{k}-T_{2}, \pi / 2}$. According to the definition of reduit, we have

$$
\begin{aligned}
v(x) & =v_{1}(x) \\
& \leq C \int_{\partial \Gamma_{-\tau, t_{k}, \pi / 2}} g^{k}\left(x, \tau\left(t_{k}\right)\right) g^{k}\left(\tau\left(t_{k}-T_{2}\right), y\right) d \nu^{k}(y), \forall x \in \Gamma_{-\tau,-t_{k}+T_{2}, \pi / 2},
\end{aligned}
$$

where we used (2.6) and the constant $C$ here is independent of $k$.

By (5.32), we have

$$
v(x) \leq C g^{k}\left(x, \tau\left(t_{k}+T_{2}\right)\right) v\left(\tau\left(t_{k}-T_{2}\right)\right) .
$$

On the other hand, by Harnack's inequality and the maximum principle, we have $u(x) \geq C^{\prime} u\left(\tau\left(t_{k}-T_{2}\right)\right) g^{k}\left(x, \tau\left(t_{k}+T_{2}\right)\right), \forall x \in \Gamma_{-\tau,-t_{k}-2 T_{2}, \pi / 2} \backslash B\left(\tau\left(t_{k}+T_{2}\right), \frac{T_{2}}{2}\right)$.

In particular, one has

$$
u(x) \geq C^{\prime} u\left(\tau\left(t_{k}-T_{2}\right)\right) g^{k}\left(x, \tau\left(t_{k}+T_{2}\right)\right), \forall x \in \Gamma_{-\tau,-t_{k}+T_{2}, \pi / 2} .
$$

Combining (5.33) and (5.34), we have

$$
\frac{v(x)}{u(x)} \leq C \frac{v\left(\tau\left(t_{k}-T_{2}\right)\right)}{u\left(\tau\left(t_{k}-T_{2}\right)\right)}
$$

where $C$ is independent of $k$. Similarly, one has

$$
C^{-1} \frac{v\left(\tau\left(t_{k}-T_{2}\right)\right)}{u\left(\tau\left(t_{k}-T_{2}\right)\right)} \leq \frac{v(x)}{u(x)} \leq C \frac{v\left(\tau\left(t_{k}-T_{2}\right)\right)}{u\left(\tau\left(t_{k}-T_{2}\right)\right)}, \forall x \in \Gamma_{-\tau,-t_{k}+T_{2}, \pi / 2} .
$$


Let $x=\tau\left(t_{0}\right)$. Then

$$
\frac{v(x)}{u(x)} \leq C^{2} \frac{v\left(\tau\left(t_{0}\right)\right)}{u\left(\left(\tau\left(t_{0}\right)\right)\right)}:=\lambda, \forall x \in \Gamma_{-\tau,-t_{k}+T_{2}, \pi / 2} .
$$

Since $\lambda$ is independent of $k$, we can let $k \rightarrow \infty$ and obtain

$$
\frac{v(x)}{u(x)} \leq \lambda, \forall x \in \widetilde{M}
$$

which implies $\operatorname{dim} C_{\xi} \leq 1$.

\subsection{Proof of Proposition 2.11.}

Proof. Let $x_{k}=\tau\left(t_{k}\right)$, where $t_{k} \rightarrow \infty$ are the barrier times. For $z_{i} \rightarrow \xi$ as $i \rightarrow \infty$, define $k_{x_{q}}^{i}:=\frac{G\left(x, z_{i}\right)}{G\left(x_{q}, z_{i}\right)}$. Then $k_{x_{q}}^{i}(x)$ is a sequence of positive harmonic functions on $\widetilde{M}$ that satisfy the normalization condition at $x_{q}: k_{x_{q}}^{i}\left(x_{q}\right)=1$. Therefore there exists a subsequence such that $\lim _{i \rightarrow \infty} k_{x_{q}}^{i}(x)=k_{x_{q}}(x)$ exists (where we still use the same index $i$ ).

For $i$ large enough, $z_{i} \in \Gamma_{\tau, t_{q}+T_{2}, \pi / 2}$ and, setting $\Gamma_{q}=\Gamma_{\tau, t_{q}-T_{2}, \pi / 2}$, Theorem 2.9 gives us that

$$
k_{x_{q}}(x) \leq C G\left(x, x_{q}\right), \forall x \in \widetilde{M} \backslash \Gamma_{q} .
$$

Equation (5.37) means that

$$
k_{x_{q}}\left(\xi^{\prime}\right)=0 \text { in the } \mathcal{L} \text {-sense, } \forall \xi^{\prime} \in \widetilde{M}(\infty) \backslash \overline{\Gamma_{q}} .
$$

Similarly we can consider the positive harmonic function normalized at $x_{0}$, i.e., $k_{x_{0}}(x)$.

By Harnack's inequality, there exists a constant $C_{q}$ such that for sufficiently large $i$ the following holds:

$$
C_{q}^{-1} G\left(x_{q}, z_{i}\right) \leq G\left(x_{0}, z_{i}\right) \leq C_{q} G\left(x_{q}, z_{i}\right) .
$$

Therefore we have

$$
\frac{G\left(x, z_{i}\right)}{G\left(x_{0}, z_{i}\right)} \leq C_{q} \frac{G\left(x, z_{i}\right)}{G\left(x_{q}, z_{i}\right)}
$$

for large $i$.

So we have

$$
k_{x_{0}}(x) \leq C_{q} k_{x_{q}}(x) \leq C_{q} G\left(x, x_{q}\right), \forall x \in \widetilde{M} \backslash \Gamma_{q} .
$$

On the other hand, by Proposition 4.6, for any $\varepsilon>0$, there is a $q \in \mathbb{N}$ such that $\Gamma_{q} \subset \Gamma_{\tau, 0, \varepsilon}$. Combining this fact and (5.39), we obtain

$$
k_{x_{0}}\left(\xi^{\prime}\right)=0 \text { in the } \mathcal{L} \text {-sense, } \forall \xi^{\prime} \in \widetilde{M}(\infty) \backslash\{\xi\} .
$$

Since $k_{x_{0}}\left(x_{0}\right)=1, k_{x_{0}}$ is a non-trivial positive harmonic function on $\widetilde{M}$, and hence is a Poisson kernel function at $\xi$. 


\section{Abundance of Martin points}

We assume in this section that $\widetilde{M}$ is the universal cover of a compact Riemannian manifold $M$ of class $C^{3}$, non-positive curvature and geodesic rank one. For each $v \in T \widetilde{M}$, we can write $v=(x, \vec{\theta})$ with $\vec{\theta} \in T_{x} \widetilde{M}$. If $\operatorname{Exp}: T \widetilde{M} \mapsto \widetilde{M}$ is the exponential map given by $\operatorname{Exp}(v)=\operatorname{Exp}_{x}(\vec{\theta})$, we always write

$$
\sigma_{v}(t)=\operatorname{Exp}_{x}(t \vec{\theta})
$$

and

$$
\varphi_{s}(v)=\left(\operatorname{Exp}_{x}(s \vec{\theta}), \frac{d\left[\operatorname{Exp}_{x}(s \vec{\theta})\right]}{d s}\right)=\left(\sigma_{v}(s), \sigma_{v}^{\prime}(s)\right) .
$$

The 1-parameter family of diffeomorphisms $\left\{\varphi_{s}\right\}$ is called the geodesic flow on $S \widetilde{M}$.

Let $\pi: S \widetilde{M} \mapsto \widetilde{M}$ be the foot-point projection. For any given $v \in S \widetilde{M}$, we consider the Busemann function

$$
\hat{b}_{v}(x)=\lim _{t \rightarrow+\infty}\left[d\left(\sigma_{v}(0), \sigma_{v}(t)\right)-d\left(x, \sigma_{v}(t)\right)\right]=\lim _{t \rightarrow+\infty}\left[t-d\left(x, \sigma_{v}(t)\right)\right],
$$

for $x \in \widetilde{M}$.

The level set $\Sigma_{v}=\hat{b}_{v}^{-1}(0)$ is called a horosphere with the inner normal vector $v$. We also let

$$
\mathcal{H}_{v}=\left\{\left(y,\left.\nabla \hat{b}_{v}\right|_{y}\right) \mid \hat{b}_{v}(y)=0\right\}
$$

be the corresponding stable leave. Clearly $\Sigma_{v}=\pi\left(\mathcal{H}_{v}\right)$ and since $M$ is of class $C^{3}, \mathcal{H}_{v}$ is a $C^{2}$-smooth embedded disc in $S \widetilde{M}$. As $v$ varies, the sets $\mathcal{H}_{v}$ form a continuous lamination of $S \widetilde{M}$ (cf. [HI], Proposition 3.1).

Furthermore, since all geodesic balls are convex, the sup-level set $\hat{b}_{v}^{-1}([c, \infty))$ is convex for all $c \in \mathbb{R}$; see [BGS].

Suppose that sectional curvatures $K$ of $\widetilde{M}$ satisfy $-1 \leq K \leq 0$. The standard Hessian comparison theorem $[\mathrm{Pe}]$ asserts that

$$
\|X\|^{2} \geq \operatorname{Hess}\left(-\hat{b}_{v}\right)(X, X) \geq 0 .
$$

Proposition 6.1. Suppose that sectional curvatures $K$ of $\widetilde{M}$ satisfy $-1 \leq K \leq$ 0 . Then for any given $\varepsilon>0$, there is an $\eta>0$ such that if $v^{\prime} \in \mathcal{H}_{v}$ satisfies $d_{\mathcal{H}_{v}}\left(v, v^{\prime}\right)<\eta$, then $d_{S \widetilde{M}}\left(\varphi_{t}(v), \varphi_{t}\left(v^{\prime}\right)\right)<\varepsilon$ for all $t \geq 0$.

Proof. If $\Omega$ is a convex subset of $\widetilde{M}$, the nearest point projection $\mathcal{P}_{\Omega}: \widetilde{M} \mapsto \Omega$ is a distance non-increasing map (see [BGS]). Consider $\Omega_{s}=\hat{b}_{v}^{-1}([s, \infty))$. Since $\Omega_{s}$ is convex, $\mathcal{P}_{\Omega_{s}}$ is a distance non-increasing map, and

$$
d_{\Sigma_{\varphi_{t}(v)}}\left(\sigma_{v}(t), \sigma_{v^{\prime}}(t)\right) \leq d_{\Sigma_{v}}\left(v, v^{\prime}\right)<\eta
$$

for all $t \geq 0$.

Recall that $\operatorname{Hess}\left(\hat{b}_{v}\right)(X, Y)=\left\langle\nabla_{X}\left(\nabla \hat{b}_{v}\right), Y\right\rangle$. Let $\Psi_{t}:[0, \eta] \mapsto \Sigma_{\varphi_{t}(v)}$ be a length-minimizing geodesic from $\sigma_{v}(t)$ to $\sigma_{v^{\prime}}(t)$ with respect to the induced metric on the horosphere $\Sigma_{\varphi_{t}(v)}=b_{v}^{-1}(t)$ of height $t$. By the fact that $\|X\|^{2} \geq$ $\operatorname{Hess}\left(-\hat{b}_{v}\right)(X, X) \geq 0$ and since $d_{\Sigma_{\varphi_{t}(v)}}\left(\sigma_{v}(t), \sigma_{v^{\prime}}(t)\right)<\eta$, we obtain, by integrating 
along the curve $\Psi_{t}$,

$$
\left\|\sigma_{v^{\prime}}^{\prime}(t)-\mathbb{P}_{\Psi_{t}}\left[\sigma_{v}^{\prime}(t)\right]\right\|=\left\|\left.\nabla \hat{b}_{v}\right|_{\sigma_{v^{\prime}}(t)}-\mathbb{P}_{\Psi_{t}}\left[\left.\nabla \hat{b}_{v}\right|_{\sigma_{v}(t)}\right]\right\| \leq \int_{\Psi_{t}}\left\|\nabla\left(\nabla \hat{b}_{v}\right)\right\|<\eta,
$$

where $\mathbb{P}_{\Psi}$ is the parallel translation along the curve $\Psi_{t}$. It follows that

$$
d_{S \widetilde{M}}\left(\varphi_{t}(v), \varphi_{t}\left(v^{\prime}\right)\right)<2 \eta
$$

This completes the proof.

Fix $x \in \widetilde{M}$ and let $\nu$ be the Patterson-Sullivan measure on $\widetilde{M}(\infty)$ associated to $x$ (see $[\mathrm{K} 1]$ ). Recall that $(\widetilde{M}(\infty) \times \widetilde{M}(\infty))^{*}$ is the set of distinct pairs of points of the geometric boundary $\widetilde{M}(\infty)$ and that the action of the covering group $\Gamma$ extends to $\widetilde{M}(\infty)$ by continuity and on $(\widetilde{M}(\infty) \times \widetilde{M}(\infty))^{*}$ by $\gamma(\eta, \xi)=(\gamma \eta, \gamma \xi)$. In [K1], $[\mathrm{K} 2]$ the following properties of $\nu$ are shown:

- 1) ([K1], Lemma 4.1) The support of the measure $\nu \times \nu$ is $(\widetilde{M}(\infty) \times \widetilde{M}(\infty))^{*}$.

- 2)([K2], Corollary 4.4) For $(\nu \times \nu)$ almost every $(\eta, \xi)$, there is a unique regular geodesic $\sigma_{\eta, \xi}$ such that $\sigma_{\eta, \xi}(-\infty)=\eta, \sigma_{\eta, \xi}(+\infty)=\xi$.

- 3)([K2], Lemma 2.4) There is a positive continuous function $F$ on $(\widetilde{M}(\infty) \times$ $\widetilde{M}(\infty))^{*}$ such that the measure $\tilde{\nu}=F(\nu \times \nu)$ is $\Gamma$-invariant.

To a vector $v \in S M$, one associates $Q(v) \in(\widetilde{M}(\infty) \times \widetilde{M}(\infty))^{*} \times \mathbb{R}$ by

$$
Q(v)=\left(\sigma_{v}(-\infty), \sigma_{v}(+\infty), b(v)\right)
$$

where $b(v)=\lim _{t \rightarrow \infty}\left(d\left(x, \sigma_{v}(t)\right)-t\right)$. The map $Q$ is a bijection from $S \widetilde{M}$ on its image. By 2), its image has full $\tilde{\nu} \times d t$ measure. The measure $\left(Q^{-1}\right)_{*}(\tilde{\nu} \times d t)$ is therefore a measure on $S \widetilde{M}$. By 3 ) it is a $\Gamma$-invariant measure. By definition, it is also invariant under the geodesic flow. It corresponds to a measure $\bar{\nu}$ on $S M$, which is invariant under the geodesic flow. By 1 ), the support of $\bar{\nu}$ is $S M$. By [K2], Theorem 4.3, the measure $\bar{\nu}$ is ergodic under the geodesic flow.

The unit sphere $S_{x} \widetilde{M}$ is transversal to the foliation $\mathcal{H}$ and to the orbits of the geodesic flow, so that a tubular neighborhood of $S_{x} \widetilde{M}$ will contain a neighborhood of the form $\bigcup_{v \in S_{x} \widetilde{M}}\left(\bigcup_{s,|s| \leq \rho} \varphi_{s} \mathcal{U}_{v}\right)$ where $\mathcal{U}_{v}$ is a neighborhood of $v$ in $\mathcal{H}_{v}$. Consider the measure $\nu_{x}$ on $S_{x} \widetilde{M}$ defined by $\nu_{x}=\left(P_{x}^{-1}\right)_{*} \nu$. On a neighborhood of $S_{x} \widetilde{M}$ of the above form, the measure $\tilde{\nu} \times d t$ has a positive density with respect to the integral over $\nu_{x}$ of positive measures with full support on $\bigcup_{s,|s| \geq \rho} \varphi_{s} \mathcal{U}_{v}$ (see [L], section 3, for the completely analogous case of negative curvature; another description of this product structure is in $[\mathrm{Gu}]$ ). This shows the following:

Proposition 6.2. Let $A$ be a Borel subset of $S_{x} \widetilde{M}$ with $\nu_{x}(A)>0$. Then, for all $\eta>0,(\tilde{\nu} \times d t)\left(\widetilde{A}_{\eta}\right)>0$, where

$$
\widetilde{A}_{\eta}=\bigcup_{v \in A}\left(\bigcup_{s,|s| \leq \eta} \varphi_{s} B^{\mathcal{H}_{v}}(v, \eta)\right)
$$

and $B^{\mathcal{H}_{v}}(v, \eta)$ is the ball of radius $\eta$ in $\mathcal{H}_{v}$ centered at $v$. 
We are now able to show that the set of directions that are not hyperbolic at $\infty$ is $\nu$ negligible. More precisely,

Proposition 6.3. There exist $h, T$ and $R$ such that, if $\mathcal{F}_{K}$ is the set of directions $v \in S_{x} \widetilde{M}$ such that $\sigma_{v}(\cdot-t)$ never admits an $(h, T, R)$ barrier for any $t \geq K$, then $\mathcal{F}_{K}$ has no interior in $S_{x} \widetilde{M}$ and $\nu_{x}\left(\mathcal{F}_{K}\right)=0$.

Proof. Recall the set $\mathcal{O}_{K}$ from Section 2. For the sake of the proof, we introduce a slightly smaller set $\mathcal{O}_{K}^{\prime}$ which is also generic and full measure, but is disjoint from $\left(\widetilde{\mathcal{F}_{K}}\right)_{\eta}$. The conclusion then follows from Proposition 6.2. Fix $\delta>0$ small.

Definition 6.4. We say that the geodesic $\sigma$ admits an $(h, T, R, \delta)$ barrier if there exist $t_{i}, i=1,2, \ldots, 6$ with $T_{1}+i \delta<t_{i+1}-t_{i}<T_{1}+R-i \delta$ and $t_{3}+T<0<t_{4}$ such that the geodesic $\sigma$ is $(h, T, \pi / 2+\delta)$-non-flat at $t_{i}$, for $i=1,2, \ldots, 6$.

As before, one can find $h, T$ and $R$ such that there is an axis that admits an $(h, T, R, \delta)$ barrier. By Proposition 4.2 the set $\mathcal{O}^{\prime}$ of $v$ such that $\sigma_{v}$ admits an $(h, T, R, \delta)$ barrier is open. Since the measure $\bar{\nu}$ is ergodic and has full support, for all positive $K$ the set $\mathcal{O}_{K}^{\prime}$ of $v \in S M$ such that the geodesic ray $\sigma_{v}([K, \infty))$ intersects $\mathcal{O}^{\prime}$ is open dense in $S M$ and has full $\bar{\nu}$ measure. By Proposition 4.2 again, we can find a number $\varepsilon>0$ such that whenever $w \in S \widetilde{M}$ is such that $\sigma_{w}$ admits an $(h, T, R, \delta)$ barrier and $d_{S \widetilde{M}}\left(w, w^{\prime}\right)<\varepsilon$, then $\sigma_{w^{\prime}}$ admits an $(h, T, R)$ barrier. Choose $\eta$ associated to $\varepsilon$ by Proposition 6.1. Now, if $v \in \mathcal{F}_{K}$ and $v^{\prime} \in$ $\bigcup_{s,|s| \leq \eta} \varphi_{s} B^{\mathcal{H}_{v}}(v, \eta)$, then $v^{\prime}$ cannot belong to $\mathcal{O}_{K}^{\prime}$ since it would mean that there is a $t>K$ such that $\sigma_{v^{\prime}}(t)$ admits an $(h, T, R, \delta)$ barrier. Since $d_{S \widetilde{M}}\left(\sigma_{v}(t), \sigma_{v^{\prime}}(t)\right)<\varepsilon$, $\sigma_{v}(t)$ would admit an $(h, T, R)$ barrier, contrarily to the definition of $\mathcal{F}_{K}$, we have shown that $\left(\widetilde{\mathcal{F}_{K}}\right)_{\eta}$ is disjoint from $\mathcal{O}_{K}^{\prime}$, an open set of full $\bar{\nu}$ measure. By Proposition $6.2, \nu_{x}\left(\mathcal{F}_{K}\right)=0$. It is also easy to see that for the same reason, $\mathcal{F}_{K}$ has no interior.

This proves the first part of Theorem 1.7, since the set of geodesics that are not hyperbolic at $\infty$ and start from $x$ is exactly the union over $K \in \mathbb{N}$ of the $\mathcal{F}_{K}$ s. For the second part, recall from the introduction the definition of a geodesic ergodic measure on $\widetilde{M}(\infty)$ :

- 1) The support of the measure $\mu \times \mu$ is $(\widetilde{M}(\infty) \times \widetilde{M}(\infty))^{*}$.

- 2) For $\mu \times \mu$ almost every $(\eta, \xi)$, there is a unique geodesic $\sigma_{\eta, \xi}$ such that $\sigma_{\eta, \xi}(-\infty)=\eta, \sigma_{\eta, \xi}(+\infty)=\xi$, and $\sigma_{\eta, \xi}$ is rank 1 .

- 3) The measure $\mu \times \mu$ is $\Gamma$-quasi-invariant and ergodic: the diagonal action of $\Gamma$ preserves the $(\mu \times \mu)$ negligible subsets of $(\widetilde{M}(\infty) \times \widetilde{M}(\infty))^{*}$, and measurable subsets of $(\widetilde{M}(\infty) \times \widetilde{M}(\infty))^{*}$ which are $\Gamma$-invariant are either negligible or conegligible.

For $(\eta, \xi) \in(\widetilde{M}(\infty) \times \widetilde{M}(\infty))^{*}$, define $N(\eta, \xi)$ as the number of times, separated by at least $4 T_{2}$, that the geodesic $\sigma_{\eta, \xi}$, if it is unique, admits an $(h, T, R)$ barrier. By property 2) above the function $N(\eta, \xi)$ is $(\mu \times \mu)$ almost everywhere well defined. Moreover, the function $N(\eta, \xi)$ clearly is $\Gamma$-invariant and therefore $(\mu \times \mu)$ almost everywhere constant. We claim that this constant cannot be a finite $K$. Indeed, we just proved that there is an open set $\mathcal{O}^{\prime \prime}$, such that for $\xi \in \mathcal{O}^{\prime \prime}$, there is a regular geodesic $\sigma_{\xi}$ with $\sigma(0)=x, \sigma(+\infty)=\xi$ and at least $K+1$ instants $t_{1}, \ldots, t_{K+1}$ with $t_{j}-t_{j+1}>4 T_{2}$, when $\sigma_{\xi}\left(\cdot-t_{j}\right)$ admits an $(h, T, R)$ barrier. For such a $\xi$, we can find, by [Ba1], a small neighborhood $O_{\xi}$ of $\sigma_{\xi}(-\infty)$ such that for $\eta \in O_{\xi}$, there is a 
unique $\sigma_{\eta, \xi}$, and it is close enough to $\sigma_{\xi}$ that we still have $N(\eta, \xi) \geq K+1$. Since $\mu \times \mu\left(\bigcup_{\xi \in \mathcal{O}^{\prime \prime}}\left(O_{\xi} \times\{\xi\}\right)\right)>0$, this is a contradiction.

So, for $(\mu \times \mu)$ almost every $(\eta, \xi), N(\eta, \xi)$ is infinite. Let $N_{+}, N_{-}, N$ be the subsets of $\{(\eta, \xi): N(\eta, \xi)=\infty\}$ where there are an infinite number of barrier times respectively only on the positive side of $\mathbb{R}$, only on the negative side or on both sides. These three sets are disjoint and $\Gamma$-invariant. Only one of them is of full measure. By Remark 2.7, the sets $N_{+}$and $N_{-}$have the same measure, which has to be 0 . Therefore, the set $N$ has full measure. In other words, $(\mu \times \mu)$ almost every geodesic is hyperbolic at $\infty$. It follows that for $\mu$ almost every $\xi \in \widetilde{M}(\infty)$, there is at least one geodesic which is asymptotic to $\xi$ and hyperbolic at $\infty$. Theorem 1.7 follows from Theorem 1.3.

\section{REFERENCES}

[An] Ancona, A., Negatively curved manifolds, elliptic operators, and the Martin boundary, Ann. Math. vol. 125 (1987), 495-536. MR0890161 (88k:58160)

[AS] Anderson, M. and Schoen, R., Positive harmonic functions on complete manifolds of negative curvature, Ann. Math. vol. 121 (1985), 429-461. MR0794369 (87a:58151)

[Ba1] Ballmann, W., Axial isometries of manifolds of non-positive curvature, Math. Ann. Vol. 259 (1982), 131-144. MR0656659 (83i:53068)

[Ba2] Ballmann, W., Nonpositively curved manifolds of higher rank. Ann. Math. vol. 122 (1985), 597-609. MR0819559 (87e:53059)

[Ba3] Ballmann, W., On the Dirichlet problem at infinity for manifolds of nonpositive curvature, Forum Math. vol.1 (1989) 201-213. MR0990144 (90j:53059)

[Ba4] Ballmann, W., The Martin Boundary of certain Hadamard Manifolds, Proceedings on Analysis and Geometry (Russian) (Novosibirsk Akademgorodok, 1999), 36-46, Izdat. Ross. Akad. Nauk Sib. Otd. Inst. Mat., Novosibirsk, 2000. MR1847509 (2002g:53043)

[BGS] Ballmann, W., Gromov, M. and Schroeder, V., Manifolds of nonpositive curvature. Progress in Mathematics, vol. 61. Birkhäuser Boston, Inc., Boston, MA, 1985. MR0823981 (87h:53050)

[BL] Ballmann, W. and Ledrappier, F., The Poisson boundary for rank one manifolds and their cocompact lattices, Forum Math. vol. 6 (1994) 301-313. MR1269841 (95b:58169)

[BS] Burns, K. and Spatzier, R. Manifolds of nonpositive curvature and their buildings. Publ. Math. IHÉS, vol 65, (1987) 35-59. MR0908215 (88g:53050)

[DoC] Do Carmo, M., Differential Geometry of Curves and Surfaces, Prentice-Hall, Inc., New Jersey, 1976. MR0394451 (52:15253)

[EO] Eberlein, P. and O'Neill, B., Visibility manifolds, Pacific J. Math. Vol. 46 (1973) 45-109. MR0336648 (49:1421)

[GJT] Guivarc'h, Y., Ji, L. and Taylor, J.C., Compactifications of symmetric spaces, Birkhäuser, Boston, 1998. MR1633171 (2000c:31006)

[Gu Gunesch, R., Precise asymptotics for the periodic orbits of the geodesic flow in negative curvature, preprint.

[HI] Heintze, E. and Im Hof, H.-C., Geometry of horospheres, J. Diff. Geom. vol. 12 (1977) 481-491. MR0512919 (80a:53051)

[K1] Knieper, G., On the asymptotic geometry of nonpositively curved manifolds, Geom. Funct. Anal., vol. 7 (1997), 755-782. MR1465601 (98h:53055)

[K2] Knieper, G., The uniqueness of the measure of maximal entropy on rank 1 manifolds, Ann. Math. vol. 148 (1998), 291-314. MR1652924 (2000b:37016)

[Ka] Kaimanovich, V. A., Boundaries of invariant Markov operators: the identification problem, Ergodic theory of $Z^{d}$ actions (Warwick, 1993-1994), 127-176, London Math. Soc. Lecture Note Ser., 228. MR1411218 (97j:31008)

[L] Ledrappier, F., Harmonic measures and Bowen-Margulis measures, Israel J. Math. vol. 71 (1990) 275-282. MR1088820 (92a:58107)

[MV] Mazzeo, R. and Vasy, A., Resolvents and Martin boundary of product spaces, Geom. Funct. Anal., vol. 12 (2002) 1018-1079. MR1937834 (2003i:58061) 
[Pe] Petersen, P., Riemannian Geometry, Springer-Verlag, GTM. Vol.171, New York 1998. MR1480173 (98m:53001)

[SY] Schoen, Y. and Yau, S.-T., Lectures on Differential Geometry, International Press, Boston, 1994. MR1333601 (97d:53001)

Department of Mathematics, University of Notre Dame, Notre Dame, Indiana 46556

E-mail address: jcao@nd.edu

School of Mathematical Sciences, Peking University, Beijing 100875, People's RePUBliC OF CHINA

E-mail address: fanhj@math.pku.edu.cn

Department of Mathematics, University of Notre Dame, Notre Dame, Indiana 46556

E-mail address: fledrapp@nd.edu 